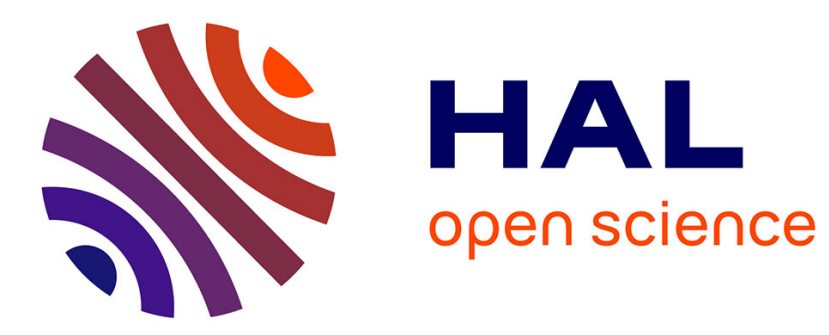

\title{
An analysis of Schwarz waveform relaxation domain decomposition methods for the imaginary-time linear Schrödinger and Gross-Pitaevskii equations
}

\author{
Xavier Antoine, Emmanuel Lorin
}

\section{- To cite this version:}

Xavier Antoine, Emmanuel Lorin. An analysis of Schwarz waveform relaxation domain decomposition methods for the imaginary-time linear Schrödinger and Gross-Pitaevskii equations. Numerische Mathematik, 2017, 137 (4), pp.923-958. 10.1007/s00211-017-0897-3 . hal-01244513

HAL Id: hal-01244513

https://hal.science/hal-01244513

Submitted on 7 Feb 2016

HAL is a multi-disciplinary open access archive for the deposit and dissemination of scientific research documents, whether they are published or not. The documents may come from teaching and research institutions in France or abroad, or from public or private research centers.
L'archive ouverte pluridisciplinaire HAL, est destinée au dépôt et à la diffusion de documents scientifiques de niveau recherche, publiés ou non, émanant des établissements d'enseignement et de recherche français ou étrangers, des laboratoires publics ou privés. 


\title{
An analysis of Schwarz waveform relaxation domain decomposition methods for the imaginary-time linear Schrödinger and Gross-Pitaevskii equations
}

\author{
X. Antoine ${ }^{\mathrm{a}, \mathrm{b}}$, E. Lorin ${ }^{\mathrm{c}, \mathrm{d}}$ \\ ${ }^{a}$ Institut Elie Cartan de Lorraine, Université de Lorraine, F-54506 Vandoeuvre-lès-Nancy Cedex, France \\ ${ }^{b}$ Inria Nancy Grand-Est/IECL - SPHINXS. \\ ${ }^{c}$ Centre de Recherches Mathématiques, Université de Montréal, Montréal, Canada, H3T 1J4 \\ ${ }^{d}$ School of Mathematics and Statistics, Carleton University, Ottawa, Canada, K1S 5B6
}

\begin{abstract}
The aim of this paper is to derive and numerically validate some asymptotic estimates of the convergence rate of Classical and Optimized Schwarz Waveform Relaxation (SWR) domain decomposition methods applied to the computation of the stationary states of the one-dimensional linear and nonlinear Schrödinger equations with a potential. Although SWR methods are currently used for efficiently solving high dimensional partial differential equations, their convergence analysis and most particularly obtaining expressions of their convergence rate remains largely open even in one dimension, except in simple cases. In this work, we tacke this problem for linear and nonlinear one-dimensional Schrödinger equations by developing techniques which can be extended to higher dimensional problems and other types of PDEs. The approach combines the method developed in [24] for the linear advection reaction diffusion equation and the theory of inhomogeneous pseudodifferential operators in conjunction with the associated symbolical asymptotic expansions. For computing the stationary states, we consider the imaginary-time formulation of the Schrödinger equation based on the Continuous Normalized Gradient Flow (CNGF) method and use a semiimplicit Euler scheme for the discretization. Some numerical results in the one-dimensional case illustrate the analysis for both the linear Schrödinger and Gross-Pitaevskii equations.
\end{abstract}

\section{Introduction}

Let us consider the following initial boundary-value problem: find the complex-valued wavefunction $u(\mathbf{x}, t)$ solution to the real-time nonlinear cubic Schrödinger equation set on $\mathbb{R}^{d}, d \geq 1$,

$$
\left\{\begin{array}{l}
i \partial_{t} u=-\triangle u+V(\mathbf{x}) u+\kappa|u|^{2} u, \mathbf{x} \in \mathbb{R}^{d}, t>0 \\
u(\mathbf{x}, 0)=u_{0}(\mathbf{x}), \mathbf{x} \in \mathbb{R}^{d}
\end{array}\right.
$$

with initial condition $u_{0}$. (Even if there is usually a $1 / 2$ coefficient in front of the kinetic operator $-\triangle$ in the first equation of system (1) for quantum mechanics (dimensionless form), we omit this coefficient here since this paper is mainly devoted to mathematical and numerical analysis questions.) The real-valued space-dependent smooth potential $V$ is positive (respectively negative) for attractive (respectively repulsive) interactions. The nonlinearity strength $\kappa$ is a real-valued

Email addresses: xavier.antoine@univ-lorraine.fr (X. Antoine), elorin@math.carleton.ca (E. Lorin) 
constant which is positive (respectively negative) for a focusing (respectively defocusing) nonlinearity. If $\kappa=0$, then we speak about the time-dependent Linear Schrödinger Equation (LSE). In the Physics literature, the first equation of system (1) is also called the Gross-Pitaevskii Equation (GPE) $[3,11,14]$ when considering Bose-Einstein Condensates (BEC) (see e.g. [35, 36]).

The computation of stationary states, e.g. ground state and excited states, is a major question in quantum physics, most particularly for BECs. Such a problem corresponds $[9,10,11,14,15,16,17]$ to computing a real number $\mu$ and a space dependent function $\phi$ which satisfies the equation

$$
\mu \phi(\mathbf{x})=-\triangle \phi(\mathbf{x})+V(\mathbf{x}) \phi(\mathbf{x})+\kappa|\phi(\mathbf{x})|^{2}, \mathbf{x} \in \mathbb{R}^{d},
$$

under the normalization constraint

$$
\|\phi\|_{L^{2}\left(\mathbb{R}^{d}\right)}^{2}:=\int_{\mathbb{R}^{d}}|\phi(\mathbf{x})|^{2} d \mathbf{x}=1 .
$$

If we define the total energy of the system as

$$
E_{\kappa}(\chi):=\int_{\mathbb{R}^{d}}|\nabla \chi(\mathbf{x})|^{2}+V(\mathbf{x})|\chi(\mathbf{x})|^{2}+\frac{\kappa}{2}|\chi(\mathbf{x})|^{4} d \mathbf{x}
$$

then a stationary state is such that

$$
E_{\kappa}(\phi):=\min _{\|\chi\|_{L^{2}\left(\mathbb{R}^{d}\right)}=1} E_{\kappa}(\chi)
$$

Once it is obtained, the eigenvalue $\mu$ (also called chemical potential) can be computed through the eigenfunction $\phi$ by using the expression

$$
\mu:=\mu_{\kappa}(\phi)=E_{\kappa}(\phi)+\frac{\kappa}{2} \int_{\mathbb{R}^{d}}|\phi(\mathbf{x})|^{4} d \mathbf{x} .
$$

Existence and uniqueness results for the minimizers corresponding to a ground state (global minimizer) or excited states (local minimizers) can be found for example in [14]. More general versions of the GPE include rotational terms, complex nonlinear (nonlocal) functions and coupled species of cold gases [3, 11, 13, 14, 37].

To numerically determine $(\mu, \phi)$, a well-known method is the so-called imaginary-time method $[9,10,11,14,15,16,17,18,20]$ which is also designated as Continuous Normalized Gradient Flow (CNGF) in the Applied Mathematics literature. It consists in solving (1) in imaginary-time, i.e. setting $t \rightarrow i t$. This transformation leads to the formulation

$$
\left\{\begin{array}{l}
\partial_{t} \phi(\mathbf{x}, t)=-\nabla_{\phi^{*}} E_{\kappa}(\phi) \\
=\triangle \phi(\mathbf{x}, t)-V(\mathbf{x}) \phi(\mathbf{x}, t)-\kappa|\phi|^{2} \phi(\mathbf{x}, t), \mathbf{x} \in \mathbb{R}^{d}, t_{n}<t<t_{n+1}, \\
\phi\left(\mathbf{x}, t_{n+1}\right):=\phi\left(\mathbf{x}, t_{n+1}^{+}\right)=\frac{\phi\left(\mathbf{x}, t_{n+1}^{-}\right)}{\left\|\phi\left(\cdot, t_{n+1}^{-}\right)\right\|_{L^{2}\left(\mathbb{R}^{d}\right)}}, \\
\phi(\mathbf{x}, t)=\phi_{0}(\mathbf{x}), \mathbf{x} \in \mathbb{R}^{d}, \text { with }\left\|\phi_{0}\right\|_{L^{2}\left(\mathbb{R}^{d}\right)}=1 .
\end{array}\right.
$$

In the above system of equations, $t_{0}:=0<t_{1}<\ldots<t_{n+1}<\ldots$ are discrete times, $\phi_{0}$ is an initial data for the time marching algorithm discretizing the projected gradient method and $\lim _{t \rightarrow t_{n}^{ \pm}} \phi(\mathbf{x}, t)=\phi\left(\mathbf{x}, t_{n}^{ \pm}\right)$. It can be proven [16] that the energy is diminishing for positive $V$ and $\kappa=0$. Let us assume now that the time discretization is uniform. We define the time step 
$\Delta t:=t_{n+1}-t_{n}$. In this paper, the chosen semi-discrete time scheme for solving the CNGF (3) is the Semi-Implicit Euler scheme (SIE) for the imaginary-time method (the associated scheme is denoted by CNGF-SIE), which reads

$$
\left\{\begin{array}{l}
\frac{\widetilde{\phi}^{n+1}-\phi^{n}}{\Delta t}-\Delta \widetilde{\phi}^{n+1}+V(\mathbf{x}) \widetilde{\phi}^{n+1}+\kappa\left|\phi^{n}\right|^{2} \widetilde{\phi}^{n+1}=0 \\
\phi^{n+1}=\frac{\widetilde{\phi}^{n+1}}{\left\|\widetilde{\phi}^{n+1}\right\|_{L^{2}\left(\mathbb{R}^{d}\right)}}, \\
\phi^{0}=\phi_{0}, \text { with }\left\|\phi^{0}\right\|_{L^{2}\left(\mathbb{R}^{d}\right)}=1 .
\end{array}\right.
$$

For the imaginary-time method (3), the semi-implicit Euler method is well-adapted. Indeed, it can be stated that the energy is then unconditionally decaying unlike e.g. the Crank-Nicolson scheme which is only energy decaying under a CFL condition [16]. Concerning the spatial discretization, various methods can be considered. For example, FFT-based pseudo-spectral methods are often used [10,11, 14, 16, 37], yielding then a highly accurate solution when combined with iterative Krylov subspace solvers $[9,10]$. Other techniques rather make use of high-order finite difference or adaptive finite element approximations [21, 22] to get accurate solutions e.g. when one needs to capture the nucleation of quantum vortices. Even if this last class of methods works correctly, considering three-dimensional problems can lead to the numerical solution of large scale linear systems which are extremely costly to solve. This is more particularly true for the CNGF-SIE scheme since a linear system must be solved at each iteration of the projected gradient technique. The aim of this paper is to contribute to understanding Domain Decomposition Methods (DDMs) for solving the CNGF system for the one-dimensional LSE and GPE. Domain decomposition methods are particularly well-adapted for the parallel solution of linear systems that appear in finite difference and finite element methods.

Among the various domain decomposition methods [23, 27], we focus our attention here on the Classical and Optimized Schwarz Waveform Relaxation (CSWR and OSWR) DDMs [1, 24, 25, 27, 28, 29, 26, 30, 31, 34]. Even if these methods have received much attention over the past years for many applications, to the best of the authors' knowledge, the first application to the Schrödinger equation can be found in [31]. The authors consider the real-time linear onedimensional Schrödinger equation with a constant potential. Well-posedness results are stated and continuous and discrete analysis of the algorithm are developed. In addition, some numerical simulations illustrate the study. Another recent paper for the Schrödinger equation is [12] where the algorithms are analyzed for a one-dimensional time-dependent linear Schrödinger equation that includes ionization and recombination by intense electric field. In [19], the authors study the numerical performance of Schwarz waveform relaxation methods for the one-dimensional dynamical solution of the LSE with a general potential, most particularly regarding their efficiency when a GPU implementation is considered. The behavior of the method shows that it can lead to fast and robust algorithms for complex linear problems. In [33], domain decomposition methods have been developed when using geometric optics and frozen gaussian approximation for computing the solution to linear Schrödinger equations beyond the semi-classical regime.

The goal of the present paper is to contribute to the fundamental understanding of Schwarz waveform relaxation DDMs for solving the Schrödinger equation. Although SWR-DDM methods are now extensively used in all kinds of high dimensional problems, the rigorous and thorough analysis of the rate of convergence remains clearly incomplete and is understood except in some simple configurations. In this work, we propose to contribute to this question by developing a 
general and rigorous strategy to analyze the convergence and the associated rate of convergence for the LSE and NLSE when computing stationary states. Even if the approach is developed on a specific application in quantum mechanics, it appears that the mathematical approach is general enough and should be applicable to other physics problems by adapting the technical proofs (for example concerning the methods related to the pseudodifferential operators).

The plan of the paper is the following. First, we develop in Section 2 some analytical estimates of the convergence rate for both CSWR and OSWR two domains decomposition methods for the linear Schrödinger (with variable potential) and the Gross-Pitaevskii equations by using the CNGF method (3). To this aim, we propose an extension of the techniques developed e.g. in $[24,31]$ to variable coefficients equations. In particular, we make an intensive use of the theory of fractional pseudodifferential operators [32] and its associated asymptotic symbolical calculus (see e.g. $[4,5,6,8]$ for some applications). Concerning the OSWR, we consider some well-adapted artificial boundary conditions derived in some previous works for the related equations in real-time $[2,5,6,7]$. We next consider in Section 3 the CNGF-SIE discretization and provide some complete and well-chosen examples to validate our analytical estimates. We also analyze the behavior of the CSWR and OSWR DDMs in this context. Finally, Section 4 concludes.

\section{Convergence of the CSWR and OSWR algorithms for the one-dimensional linear case with a potential $V(x)$}

\subsection{Preliminaries related to pseudodifferential operators}

Before estimating the convergence rate of the Schwarz waveform relaxation algorithms for the one-dimensional $(d=1)$ imaginary-time LSE with a variable potential, let us first introduce some notions about pseudodifferential operators and microlocal analysis.

We define a pseudodifferential operator $\mathcal{A}\left(x, t, \partial_{t}\right):=\mathrm{Op}(a)$ in the Fourier space through its symbol $a(x, t, \tau)$

$$
\begin{aligned}
\mathcal{A}\left(x, t, \partial_{t}\right) u(x, t) & =\mathcal{F}_{t}^{-1}(a(x, t, \tau) \hat{u}(x, \tau)) \\
& =\int_{\mathbb{R}} a(x, t, \tau) \mathcal{F}_{t}(u)(x, \tau) e^{i t \tau} \mathrm{d} \tau:=\operatorname{Op}(a) u(x, t),
\end{aligned}
$$

where the Fourier transform in time $\mathcal{F}_{t}$ is

$$
\mathcal{F}_{t}(u)(x, \tau)=\hat{u}(x, \tau)=\frac{1}{2 \pi} \int_{\mathbb{R}} u(x, t) e^{-i t \tau} \mathrm{d} t .
$$

In this paper, we use the inhomogeneous pseudodifferential operator calculus introduced in [32] and applied in $[4,5,6,8]$ to the design of TBCs/ABCs. Since all the details cannot be included here, we only develop the necessary material to understand the paper. Let us consider a real number $\alpha$ and an open subset $\Xi$ of $\mathbb{R}$. The symbol class $\mathbb{S}^{\alpha}(\Xi \times \Xi)$ is defined as the linear space of $\mathcal{C}^{\infty}$ functions $a(x, t, \tau)$ in $\Xi \times \Xi \times \mathbb{R}$ such that for any set $K \subseteq \Xi \times \Xi$, for all indices $\beta, \delta, \gamma$, there exists a positive constant $C_{\beta, \delta, \gamma}(K)$ such that the following inequality holds

$$
(\forall(x, t) \in K)(\forall \tau \in \mathbb{R}) \quad\left|\partial_{\tau}^{\beta} \partial_{t}^{\delta} \partial_{x}^{\gamma} a(x, t, \tau)\right| \leq C_{\beta, \delta, \gamma}(K)\left(1+|\tau|^{2}\right)^{\alpha-\beta} .
$$

A function $f$ is said to be inhomogeneous of degree $m$ if it satisfies the relation: $f(x, t, \mu \tau)=$ $\mu^{m} f(x, t, \tau), \forall \mu>0$. According to this definition, a pseudodifferential operator $\mathcal{A}=\mathcal{A}\left(x, t, \partial_{t}\right)$ is 
inhomogeneous and classical of order $M$, for $M \in \mathbb{Z} / 2$, if its total symbol $a=\sigma(\mathcal{A})$ admits the following asymptotic expansion

$$
a(x, t, \tau) \sim \sum_{j=0}^{+\infty} a_{M-j / 2}(x, t, \tau)
$$

where $a_{M-j / 2}$ is an inhomogeneous function of degree $M-j / 2$, for $j \in \mathbb{N}$. In the above expression, $\sim$ means that we have the relation

$$
\forall \widetilde{m} \in \mathbb{N}, \quad a-\sum_{j=0}^{\widetilde{m}} a_{M-j / 2} \in \mathbb{S}^{M-(\widetilde{m}+1) / 2} .
$$

If $a$ is a symbol that satisfies the above expansion, then it will be denoted by $a \in \mathbb{S}_{S}^{M}$ and the corresponding pseudodifferential operator $\mathcal{A}=O p(a)$ is an element of the class $\operatorname{OPS}_{S}^{M}$. Since we are using the above pseudodifferential operator theory, we need to assume that $V$ is a smooth potential. In addition, in the linear case and because $V$ does not depend on $t$, the time variable $t$ is not needed in the sequel. However, if this would be the case, the technique extends to the calculations performed in this paper.

Let us now define the time-dependent one-dimensional Schrödinger operator $P$ as

$$
P\left(x, t, \partial_{x}, \partial_{t}\right)=i \partial_{t}+\partial_{x}^{2}-V(x)
$$

Then, the following proposition holds [6].

Proposition 2.1. We have the Nirenberg-like factorization

$$
P\left(x, t, \partial_{x}, \partial_{t}\right)=\left(\partial_{x}+i \Lambda^{-}\right)\left(\partial_{x}+i \Lambda^{+}\right)+\mathcal{R},
$$

where $\mathcal{R} \in O P S^{-\infty}$ is a smoothing pseudodifferential operator. The operators $\Lambda^{ \pm}$are pseudodifferential operators of order $1 / 2$ (in time) and order zero in $x$. Furthermore, their total symbols $\lambda^{ \pm}:=\sigma\left(\Lambda^{ \pm}\right)$can be expanded in $S_{S}^{1 / 2}$ as

$$
\lambda^{ \pm} \sim \sum_{j=0}^{+\infty} \lambda_{1 / 2-j / 2}^{ \pm}
$$

where $\lambda_{1 / 2-j / 2}^{ \pm}$are symbols corresponding to operators of order $1 / 2-j / 2$.

Practically, an approximation of $\Lambda^{ \pm}$can be obtained through the computation of a finite number of elementary inhomogeneous symbols. More precisely, one gets the proposition [6].

Proposition 2.2. Let us fix the principal symbol to

$$
\lambda_{1 / 2}^{ \pm}=\mp \sqrt{-\tau+V}
$$

Then, the next symbols are given by

$$
\lambda_{0}^{ \pm}=0, \quad \lambda_{-1 / 2}^{ \pm}=0 \quad \text { and } \quad \lambda_{-1}^{ \pm}=\mp \frac{i}{4-\tau+V}
$$


The proof of Propositions 2.1 and 2.2 is obtained by a constructive process based on the following recursive formulae. Let us fix $\lambda_{1 / 2}^{+}$and $\lambda_{0}^{+}$by the expressions given in proposition 2.2. Then, it can be proved [6] that, for $j \in \mathbb{N}^{*}$,

$$
\lambda_{-j / 2}^{+}=\frac{1}{2 \lambda_{1 / 2}^{+}}\left(-i \partial_{x} \lambda_{1 / 2-j / 2}^{+}-\sum_{k=1}^{j} \lambda_{-j / 2+k / 2}^{+} \lambda_{1 / 2-k / 2}^{+}\right),
$$

and $\lambda_{-j / 2}^{+}=\lambda_{-j / 2}^{-}$, for $j=-1 / 2,0,1 / 2 \ldots$ Based on this formula, we get

Proposition 2.3. Let us define the symbol class $\mathbb{S}_{S}^{1 / 2}$ by

$$
\begin{aligned}
\mathbb{S}_{S}^{1 / 2}:=\{a \sim & \sum_{j=0}^{+\infty} a_{1 / 2-j / 2} \in S_{S}^{1 / 2} \text { such that: } a_{1 / 2-j / 2}(x, \tau):= \\
& \left.\frac{1}{\left(\lambda_{1 / 2}^{+}\right)^{j-1}} \sum_{\ell=0}^{L_{j}} \frac{\mathcal{F}_{\ell}^{V, 1 / 2-j / 2}}{\left(\lambda_{1 / 2}^{+}\right)^{\ell}} \text {, with } \mathcal{F}_{\ell}^{V, 1 / 2-j / 2} \in \mathcal{C}^{\infty}(\mathbb{R} ; \mathbb{R}), L_{j} \in \mathbb{N}\right\},
\end{aligned}
$$

and the associated class of pseudodifferential operators $O P \mathbb{S}_{S}^{1 / 2}$. In $(13), \mathcal{F}_{\ell}^{V, 1 / 2-j / 2}$ are smooth functions depending on $x$ and $V$. Then, $\Lambda^{ \pm}$are in $O P \mathbb{S}_{S}^{1 / 2}$ and, for each $j \in \mathbb{N}$, there exist some regular functions $\left\{\mathcal{F}_{\ell}^{V, 1 / 2-j / 2}\right\}_{\ell=0}^{L_{j}}$ such that

$$
\lambda_{1 / 2-j / 2}^{+}=-\lambda_{1 / 2-j / 2}^{-}=\frac{1}{\left(\lambda_{1 / 2}^{+}\right)^{j-1}} \sum_{\ell=0}^{L_{j}} \frac{\mathcal{F}_{\ell}^{V, 1 / 2-j / 2}}{\left(\lambda_{1 / 2}^{+}\right)^{\ell}} .
$$

Proof. For $j=0$, we trivially have

$$
\lambda_{1 / 2}^{+}=-\lambda_{1 / 2}^{-}=\frac{\mathcal{F}_{0}^{V, 1 / 2}}{\left(\lambda_{1 / 2}^{+}\right)^{-1}},
$$

with $\mathcal{F}_{0}^{V, 1 / 2}=1$ and $L_{0}=1$. Assume now that, for $0 \leq k \leq j$, with $k \in \mathbb{N}$, we have

$$
\lambda_{1 / 2-k / 2}^{ \pm}=\frac{1}{\left(\lambda_{1 / 2}^{+}\right)^{k-1}} \sum_{\ell=0}^{L_{k}} \frac{\mathcal{F}_{\ell}^{V, 1 / 2-k / 2}}{\left(\lambda_{1 / 2}^{+}\right)^{\ell}} .
$$

We need to prove by induction that the expression (14) holds for $j+1$. First, from (16), a direct calculation leads to

$$
\begin{aligned}
& \partial_{x} \lambda_{1-j / 2}^{+}=\partial_{x}\left(\sum_{\ell=0}^{L_{j}} \frac{\mathcal{F}_{\ell}^{V, 1 / 2-j / 2}}{\left(\lambda_{1 / 2}^{+}\right)^{j+\ell-1}}\right)= \\
& \sum_{\ell=0}^{L_{j}}\left(\frac{(1-j-\ell)}{2} \partial_{x} V \frac{\mathcal{F}_{\ell}^{V, 1 / 2-j / 2}}{\left(\lambda_{1 / 2}^{+}\right)^{j+\ell}}+\frac{\partial_{x} \mathcal{F}_{\ell}^{V, 1 / 2-j / 2}}{\left(\lambda_{1 / 2}^{+}\right)^{j+\ell-1}}\right)=\frac{1}{\left(\lambda_{1 / 2}^{+}\right)^{j-1}} \sum_{\ell=0}^{L_{j}} \frac{\mathcal{G}_{\ell}^{V, 1 / 2-j / 2}}{\left(\lambda_{1 / 2}^{+}\right)^{\ell}},
\end{aligned}
$$

for some given functions $\mathcal{G}_{\ell}^{V, 1 / 2-j / 2}$. Now, we also have

$$
\lambda_{-j / 2+k / 2}^{+}=\lambda_{1 / 2-(j-k+1) / 2}^{+}=\frac{1}{\left(\lambda_{1 / 2}^{+}\right)^{j-k}} \sum_{\ell=0}^{L_{j-k+1}} \frac{\mathcal{F}_{\ell}^{V,(k-j) / 2}}{\left(\lambda_{1 / 2}^{+}\right)^{\ell}} .
$$


As a consequence, we deduce that

$$
\begin{aligned}
& \lambda_{1 / 2-k / 2}^{+} \lambda_{-j / 2+k / 2}^{+}=\left(\frac{1}{\left(\lambda_{1 / 2}^{+}\right)^{k-1}} \sum_{\ell=0}^{L_{k}} \frac{\mathcal{F}_{\ell}^{V,(1-k) / 2}}{\left(\lambda_{1 / 2}^{+}\right)^{\ell}}\right)\left(\frac{1}{\left(\lambda_{1 / 2}^{+}\right)^{j-k}} \sum_{\ell=0}^{L_{j-k+1}} \frac{\mathcal{F}_{\ell}^{V,(k-j) / 2}}{\left(\lambda_{1 / 2}^{+}\right)^{\ell}}\right) \\
& =\frac{1}{\left(\lambda_{1 / 2}^{+}\right)^{j-1}}\left(\sum_{\ell=0}^{L_{k}} \frac{\mathcal{F}_{\ell}^{V,(1-k) / 2}}{\left(\lambda_{1 / 2}^{+}\right)^{\ell}}\right)\left(\sum_{\ell=0}^{L_{j-k+1}} \frac{\mathcal{F}_{\ell}^{V,(k-j) / 2}}{\left(\lambda_{1 / 2}^{+}\right)^{\ell}}\right)=\frac{1}{\left(\lambda_{1 / 2}^{+}\right)^{j-1}} \sum_{\ell=0}^{L_{k}+L_{j-l+1}} \frac{\mathcal{H}_{\ell}^{V,(j, k)}}{\left(\lambda_{1 / 2}^{+}\right)^{\ell}}
\end{aligned}
$$

for some functions $\mathcal{H}_{\ell}^{V,(j, k)}$ which can be expressed thanks to $\mathcal{F}_{\ell}^{V,(1-k) / 2}$ and $\mathcal{F}_{\ell}^{V,(k-j) / 2}$. Now, looking at (12), we see that, by summing up the different expressions related to (17) and (19), we have

$$
\lambda_{-j / 2}^{+}=\frac{1}{\left(\lambda_{1 / 2}^{+}\right)^{j}} \sum_{\ell=0}^{L_{j+1}} \frac{\mathcal{F}_{\ell}^{V,-j / 2}}{\left(\lambda_{1 / 2}^{+}\right)^{\ell}},
$$

showing that the result holds for the index $j+1$. The functions $\mathcal{F}_{\ell}^{V,-j / 2}$ and the index $L_{j+1}$ can be deduced from the calculations if required.

Proving that $\lambda_{1 / 2-j / 2}^{+}=-\lambda_{1 / 2-j / 2}^{-}$is a direct consequence of the results stated in [6] for Proposition 2.1.

\subsection{Asymptotic estimates of the contraction factor of the CSWR algorithm}

The real-time operator $P$ defined by (7) becomes a heat-like operator in imaginary-time following the first equation of system (3)

$$
P=\partial_{t}-\partial_{x}^{2}+V(x)
$$

Assuming that $\phi^{ \pm,(0)}(\mp \epsilon / 2, \cdot)$ are two given functions, the CSWR algorithm at iteration $k \geq 1$ reads as follows

$$
\begin{cases}P \phi^{ \pm,(k)} & =0, \text { in } \Omega_{\epsilon}^{ \pm} \times \mathbb{R}_{+}^{*}, \\ \phi^{ \pm,(k)}(\cdot, 0) & =\phi_{0}^{ \pm}, \text {in } \Omega_{\epsilon}^{ \pm} \\ \phi^{ \pm,(k)}( \pm \epsilon / 2, \cdot) & =\phi^{\mp,(k-1)}( \pm \epsilon / 2, \cdot), \text { in } \mathbb{R}_{+}^{*}\end{cases}
$$

where $\phi_{0}^{ \pm}$denotes the restriction of $\phi_{0 \mid \Omega_{\epsilon}^{ \pm}}$to $\Omega_{\epsilon}^{ \pm}$, with $\Omega_{\epsilon}^{+}=(-\infty, \epsilon / 2), \Omega_{\epsilon}^{-}=(-\epsilon / 2,+\infty)$ and $\varepsilon>0$ is the (small) size of the overlapping region. Working with the error equations, i.e. $e_{P}^{C, \pm}$ corresponds to $\phi^{ \pm}$for CSWR, we have in $\Omega_{\epsilon}^{ \pm}$

$$
P \cdot e_{P}^{C, \pm}=0 \text { in } \Omega_{\epsilon}^{ \pm} \times \mathbb{R}_{+}^{*}, \quad e_{P}^{C, \pm}( \pm \epsilon / 2, t)=h_{\epsilon}^{ \pm}(t) \text { at }\{ \pm \epsilon / 2\} \times \mathbb{R}_{+}^{*},
$$

where $P$ is given by (21). We use the index $P$ for $e_{P}^{C, \pm}$ to specify to which operator the error is associated to, and the exponent $C$ stands for the CSWR algorithm (the OSWR algorithm will be introduced later with an exponent $O$ ). In the following, some other approximate errors will also be used when the potential $V$ is variable. The time-dependent functions $h_{\epsilon}^{ \pm}$are now assumed to be given. To shorten the notations in what follows, $h_{\epsilon}^{ \pm}$also denotes the extension of $h_{\epsilon}^{ \pm}$to all $\mathbb{R}$ which is null on $\mathbb{R}_{-}$. As proposed in [24], we want to determine the contraction factor $C_{P, \epsilon}^{C}$ of $\mathcal{G}_{P}^{C 2}$ (setting $\mathcal{G}_{P}^{C 2}:=\mathcal{G}_{P}^{C} \circ \mathcal{G}_{P}^{C}$ ), where the mapping $\mathcal{G}_{P}^{C}$ is defined by

$$
\mathcal{G}_{P}^{C}:\left\langle h_{\epsilon}^{+}, h_{\epsilon}^{-}\right\rangle \mapsto\left\langle e_{P}^{C,-}(\epsilon / 2, \cdot), e_{P}^{C,+}(-\epsilon / 2, \cdot)\right\rangle .
$$


To prove that $\mathcal{G}_{P}^{C 2}$ is a contraction, we solve $(23)$ in the $(x, \tau)$-coordinates in an exact way for $V=0$. For $V \neq 0$ (in fact, for a non constant potential $V$ ), we estimate the rate of convergence through approximations.

Let us first start by assuming that $V=0$ (the extension to a constant $V$ remains valid). According to [24], for a fixed time $T, \mathcal{G}_{P}^{C}$ is defined to $H_{0}^{3 / 4}(0, T)=\left\{\phi \in H^{3 / 4}(0, T): \phi(0)=0\right\}$. Let us characterize the part of the error $e_{P}^{C,+}$ (respectively $e_{P}^{C,-}$ ) which is a traveling wave in the overlapping region related to $\Omega_{\varepsilon}^{+}$(respectively $\Omega_{\varepsilon}^{-}$) and transmitted to the left (respectively right) domain $\mathbb{R} / \overline{\Omega_{\varepsilon}^{+}}$(respectively $\mathbb{R} / \overline{\Omega_{\varepsilon}^{-}}$). To this aim, one introduces the system of equations

$$
\left\{\begin{array}{l}
\left(\partial_{x}+i \Lambda^{\mp}\right) \cdot e_{\Lambda}^{C, \pm}=0, \text { in } \Omega_{\varepsilon}^{ \pm} \\
e_{\Lambda}^{C, \pm}( \pm \epsilon / 2, t)=h_{\epsilon}^{ \pm}(t) \text { at }\{ \pm \epsilon / 2\} \times \mathbb{R}
\end{array}\right.
$$

For $V=0$, the solution of the first equation of system (25) can be made explicitly and exactly through the Fourier transform $\mathcal{F}_{t}$ in the $t$-direction (meaning at the symbol level). As we see below, if $V \neq 0$, this leads to an explicit but approximate solution. The exact solution to system $(25)$ is given in the $(x, \tau)$-space by

$$
\hat{e}_{\Lambda}^{C, \pm}(x, \tau)=\hat{h}_{\epsilon}^{ \pm}(\tau) \exp \left(-i \int_{ \pm \epsilon / 2}^{x} \lambda^{\mp}(\tau) d y\right) .
$$

In addition, since we need to use the symbols for the imaginary-time equation, we obtain the correct symbols for (21) through the symbols for (7) but with the following modifications: $t \rightarrow i t$ and $\tau \rightarrow i \tau$. As a consequence, the application of Proposition 2.2 for $V=0$ leads to: $\lambda^{ \pm}(\tau)=$ $\lambda_{1 / 2}^{ \pm}(\tau)=\mp \sqrt{-i \tau}=\mp e^{i \pi / 4} \sqrt{-\tau}$. If we define

$$
\mathcal{G}_{\Lambda}^{C}:\left\langle h_{\epsilon}^{+}, h_{\epsilon}^{-}\right\rangle \mapsto\left\langle e_{\Lambda}^{C,-}(\epsilon / 2, \cdot), e_{\Lambda}^{C,+}(-\epsilon / 2, \cdot)\right\rangle,
$$

we have the equalities

$$
\begin{aligned}
& \mathcal{F}_{t}\left(\mathcal{G}_{\Lambda}^{C 2}\left\langle h_{\epsilon}^{+}, h_{\epsilon}^{-}\right\rangle\right) \\
& =\left\langle\exp \left(i \int_{-\epsilon / 2}^{\epsilon / 2}\left(\lambda^{-}(\tau)-\lambda^{+}(\tau)\right) d y\right) \hat{h}_{\epsilon}^{+}, \exp \left(i \int_{-\epsilon / 2}^{\epsilon / 2}\left(\lambda^{-}(\tau)-\lambda^{+}(\tau)\right) d y\right) \hat{h}_{\epsilon}^{-}\right\rangle \\
& =\exp \left(-2 i \epsilon \lambda^{+}(\tau)\right)\left\langle\hat{h}_{\epsilon}^{+}, \hat{h}_{\epsilon}^{-}\right\rangle .
\end{aligned}
$$

Following for instance [24] (and for a constant potential $V$ ), we deduce that the contraction factors $C_{\Lambda, \epsilon}^{C}$ of $\mathcal{G}_{\Lambda}^{C 2}$ and $C_{P, \epsilon}^{C}$ of $\mathcal{G}_{P}^{C 2}$ are the same and that

$$
C_{P, \epsilon}^{C}=C_{\Lambda, \epsilon}^{C}=\sup _{\tau \in \mathcal{H}_{\tau}} L_{\Lambda, \epsilon}^{C}(\tau)
$$

where

$$
L_{\Lambda, \epsilon}^{C}(\tau)=\left|\exp \left(-2 i \epsilon \lambda^{+}(\tau)\right)\right|=\exp (-\epsilon \sqrt{-2 \tau}) .
$$

In the above expression, $\mathcal{H}_{\tau}$ designates the hyperbolic zone $\{\tau \in \mathbb{R}: \tau<0\}$. We can then expect a fast convergence of the DDM at high frequency and/or for a large enough overlapping region of 
size $\epsilon$. Note that the above approach is valid at any frequency, although the convergence will be naturally much slower for low-frequency waves. Without overlap $(\epsilon=0)$, the CSWR algorithm diverges.

Let us now consider the general case with a potential $V$. Then, the hyperbolic zone is defined by $\{\tau \in \mathbb{R}: \tau<V\}$. As above, we need to characterize the error that travels from one domain to the other. However, for a general potential $V$, this cannot be made exactly because of the scattering effects related to the action of $V$. Therefore, we still consider the system

$$
\left\{\begin{array}{l}
\left(\partial_{x}+i \Lambda^{\mp}\right) \cdot e_{\Lambda}^{C, \pm}=0, \text { in } \Omega_{\varepsilon}^{ \pm} \\
e_{\Lambda}^{C, \pm}( \pm \epsilon / 2, t)=h_{\epsilon}^{ \pm}(t) \text { at }\{ \pm \epsilon / 2\} \times \mathbb{R}
\end{array}\right.
$$

but $e_{\Lambda}^{C,+}$ (respectively $e_{\Lambda}^{C,-}$ ) must be understood as the part of $e_{P}^{C,+}$ (respectively $e_{P}^{C,-}$ ) which travels to the right (respectively left). As a consequence, the computation of $e_{\Lambda}^{C, \pm}$ provides an approximation of $e_{P}^{C, \pm}$ solution to $P \cdot e_{P}^{C, \pm}=0$. We approximate $C_{P, \varepsilon}^{C}$ which is the contraction factor of $\mathcal{G}_{P}^{C 2}$ by $C_{\Lambda, \varepsilon}^{C}$ for $\mathcal{G}_{\Lambda}^{C 2}$

$$
C_{P, \varepsilon}^{C} \approx C_{\Lambda, \varepsilon}^{C}
$$

For solving (28), let us consider the equation at the symbol level, i.e.

$$
\left\{\begin{array}{l}
\left(\partial_{x}+i \lambda^{\mp}(x, \tau)\right) \hat{e}_{\Lambda}^{C, \pm}(x, \tau)=0, \text { in } \Omega_{\varepsilon}^{ \pm}, \\
\hat{e}_{\Lambda}^{C, \pm}( \pm \epsilon / 2, \tau)=\hat{h}_{\epsilon}^{ \pm}(\tau) \text { at }\{ \pm \epsilon / 2\} \times \mathbb{R} .
\end{array}\right.
$$

A direct calculation shows that

$$
\hat{e}_{\Lambda}^{C, \pm}(x, \tau)=\hat{h}_{\epsilon}^{ \pm}(\tau) \exp \left(-i \int_{ \pm \epsilon / 2}^{x} \lambda^{\mp}(y, \tau) d y\right) .
$$

The computation of the contraction factor $C_{\Lambda, \epsilon}^{C}$ of the associated mapping $\mathcal{G}_{\Lambda}^{C 2}$ requires the knowledge of the total symbols $\lambda^{ \pm}$. For a general potential $V$, this is generally impossible. However, as seen in Section 2.1, we have access to some asymptotic expansions $\left\{\lambda_{1 / 2-j / 2}^{ \pm}\right\}_{j=0}^{+\infty}$ of $\lambda^{ \pm}$. To get an estimate, we first expand $\lambda^{ \pm}$asymptotically as the sum of inhomogeneous symbols

$$
\lambda^{ \pm} \approx \sum_{j=0}^{ \pm \infty} \lambda_{1 / 2-j / 2}^{ \pm}
$$

and then we truncate up to the $(p+1)$ first terms

$$
\lambda^{ \pm} \approx \lambda^{ \pm, p}=\sum_{j=0}^{p} \lambda_{1 / 2-j / 2}^{ \pm}
$$

as proposed in [6]. This means that the approximate convergence rates are

$$
C_{P, \epsilon}^{C} \approx C_{\Lambda, \epsilon}^{C} \approx C_{\epsilon}^{C, p}:=\sup _{\tau \in \mathcal{H}_{\tau}} L_{\epsilon}^{C, p}(\tau)
$$

with

$$
L_{\epsilon}^{C, p}(\tau)=\left|\exp \left(i \int_{-\epsilon / 2}^{\epsilon / 2}\left(\lambda^{-, p}(y, \tau)-\lambda^{+, p}(y, \tau)\right) d y\right)\right|
$$


Let us recall now that if one chooses the principal symbol $\lambda_{1 / 2}^{ \pm}=\mp \sqrt{-\tau+V}$, then ones gets (see Section 2.1)

$$
\lambda^{-, p}=-\lambda^{+, p},
$$

implying that (32) becomes

$$
L_{\epsilon}^{C, p}(\tau)=\left|\exp \left(-2 i \int_{-\epsilon / 2}^{\epsilon / 2} \lambda^{+, p}(y, \tau) d y\right)\right|
$$

Let us remark that (33) does not hold for $\lambda_{1 / 2}^{ \pm}=\mp \sqrt{-\tau}$ if $V \neq 0[6]$.

A third approximation step consists in developing each symbol $\lambda_{1 / 2-j / 2}^{ \pm}, j=0, \ldots, p$, according to the small parameter $1 /|\tau|$ (high-frequency regime). More precisely, for each symbol $\lambda_{1 / 2-j / 2}^{ \pm}$, we consider its Taylor's expansion $\left(\lambda_{1 / 2-j / 2}^{ \pm}\right)_{(1-p) / 2}$ up to the order $1 /|\tau|^{(p-1) / 2}$

$$
\lambda^{ \pm, p} \approx \widetilde{\lambda}^{ \pm, p}=\sum_{j=0}^{p}\left(\lambda_{1 / 2-j / 2}^{ \pm}\right)_{(1-p) / 2} .
$$

We then define

$$
\widetilde{L}_{\epsilon}^{C, p}(\tau)=\left|\exp \left(-2 i \int_{-\epsilon / 2}^{\epsilon / 2} \widetilde{\lambda}^{+, p}(y, \tau) d y\right)\right|,
$$

and the associated high-frequency asymptotic convergence rate $\widetilde{C}_{\epsilon}^{C, p}$ such that

$$
C_{P, \epsilon}^{C} \approx C_{\Lambda, \epsilon}^{C} \approx \widetilde{C}_{\epsilon}^{C, p}:=\sup _{\tau \in \mathcal{H}_{\tau}} \widetilde{L}_{\epsilon}^{C, p}(\tau)
$$

Let us set

$$
\begin{aligned}
& L_{\epsilon, 1 / 2-j / 2}(\tau)=\left|\exp \left(-2 i \int_{-\epsilon / 2}^{\epsilon / 2} \lambda_{1 / 2-j / 2}^{+}(y, \tau) d y\right)\right| \\
& \widetilde{L}_{\epsilon, 1 / 2-j / 2}^{p}(\tau)=\left|\exp \left(-2 i \int_{-\epsilon / 2}^{\epsilon / 2}\left(\lambda_{1 / 2-j / 2}^{+}\right)_{(1-p) / 2}(y, \tau) d y\right)\right| .
\end{aligned}
$$

Then, we trivially have

$$
L_{\epsilon}^{C, p}=\prod_{j=0}^{p} L_{\epsilon, 1 / 2-j / 2} \text { and } \widetilde{L}_{\epsilon}^{C, p}=\prod_{j=0}^{p} \widetilde{L}_{\epsilon, 1 / 2-j / 2}^{p} .
$$

This means that the elementary contribution of each inhomogeneous symbol and its approximate Taylorized symbol to the convergence rate can be studied separately, the global contribution being obtained by a simple multiplication. Based on these remarks, we now state some estimates of the rate of convergence of the CSWR algorithm for a general potential $V$.

Theorem 2.1. Let $V$ be a smooth potential and let us assume that the symbols are defined as in Proposition 2.2. An asymptotic estimate of the contraction factor of the mapping $\mathcal{G}_{P}^{C 2}$ defined by (24), for the CSWR algorithm (22), is given by

$$
C_{P, \epsilon}^{C} \approx C_{\epsilon}^{C, 3}=\sup _{\tau \in \mathcal{H}_{\tau}} L_{\epsilon}^{C, 3}(\tau),
$$


for $|\tau| \gg 1$, where $\mathcal{H}_{\tau}$ is the hyperbolic zone and

$$
L_{\epsilon}^{C}(\tau) \approx L_{\epsilon}^{C, 3}(\tau):=\left|\left(\frac{\tau-i V(-\epsilon / 2)}{\tau-i V(+\epsilon / 2)}\right)^{1 / 2} \| \exp \left(-2 e^{-i \pi / 4} \int_{-\epsilon / 2}^{\epsilon / 2} \sqrt{-\tau+i V(y)} d y\right)\right| .
$$

In addition, one also gets the following approximation when the symbols are Taylorized

$$
C_{P, \epsilon}^{C} \approx \widetilde{C}_{\epsilon}^{C, 3}=\sup _{\tau \in \mathcal{H}_{\tau}} \widetilde{L}_{\epsilon}^{C, 3}(\tau)
$$

with

$$
L_{\epsilon}^{C}(\tau) \approx \widetilde{L}_{\epsilon}^{C, 3}(\tau):=\exp \left(-\epsilon \sqrt{-2 \tau}-\frac{1}{\sqrt{-2 \tau}} \int_{-\epsilon / 2}^{\epsilon / 2} V(y) d y\right) .
$$

Let us remark that the potential has no effect here if it is odd. In the case of a positive potential which confines the solution into the domain (standard situation for the GPE), then the convergence rate is improved. Again, in the non overlapping case, the iterative method diverges.

Proof. From (30), we have

$$
\hat{e}_{\Lambda}^{C, \pm}(x, \tau)=\hat{h}_{\epsilon}^{ \pm}(\tau) \exp \left(-i \int_{ \pm \epsilon / 2}^{x} \lambda^{\mp}(y, \tau) d y\right) .
$$

This implies that we have the approximation

$$
\mathcal{F}_{t}\left(\mathcal{G}_{P}^{C} \circ \mathcal{G}_{P}^{C}\left\langle h_{\epsilon}^{+}, h_{\epsilon}^{-}\right\rangle\right) \approx \exp \left(i \int_{-\epsilon / 2}^{\epsilon / 2}\left(\lambda^{-}(y, \tau)-\lambda^{+}(y, \tau)\right) d y\right)\left\langle\hat{h}_{\epsilon}^{+}, \hat{h}_{\epsilon}^{-}\right\rangle .
$$

By using Proposition 2.2 for the imaginary-time equation, one gets

$$
\lambda_{1 / 2}^{ \pm}(x, \tau)=\mp e^{i \pi / 4} \sqrt{-\tau+i V(x)}, \quad \lambda_{-1}^{ \pm}(x, \tau)=\mp \frac{1}{4} \frac{V^{\prime}(x)}{\tau-i V(x)},
$$

$\lambda_{0}^{ \pm}=0$ and $\lambda_{-1 / 2}^{ \pm}(x, \tau)=0$. A direct computation leads to

$$
\begin{aligned}
& L_{\epsilon, 1 / 2}(\tau)=\left|\exp \left(-2 e^{-i \pi / 4} \int_{-\epsilon / 2}^{\epsilon / 2} \sqrt{-\tau+i V(y)} d y\right)\right|, \\
& L_{\epsilon,-1}(\tau)=\left|\exp \left(\frac{i}{2} \int_{-\epsilon / 2}^{\epsilon / 2} \frac{V^{\prime}(y)}{\tau-i V(y)} d y\right)\right|,
\end{aligned}
$$

and $L_{\epsilon, 0}=L_{\epsilon,-1 / 2}=1$. Some calculations show that

$$
L_{\epsilon,-1}(\tau)=\left|\left(\frac{\tau-i V(-\epsilon / 2)}{\tau-i V(+\epsilon / 2)}\right)^{1 / 2}\right|
$$

As a consequence, one gets

$$
L_{\epsilon}^{C, p}(\tau)=\left|\left(\frac{\tau-i V(-\epsilon / 2)}{\tau-i V(+\epsilon / 2)}\right)^{1 / 2} \| \exp \left(-2 e^{-i \pi / 4} \int_{-\epsilon / 2}^{\epsilon / 2} \sqrt{-\tau+i V(y)} d y\right)\right| .
$$


Let us note that computing the real part of $\lambda_{1 / 2}^{+}$is possible through

$$
\Re\left(-2 e^{-i \pi / 4} \sqrt{-\tau+i V}\right)=\left(a+\frac{V}{2 a}\right), \text { with } a=\left(\left(-\tau+\left(\tau^{2}+V^{2}\right)^{1 / 2}\right)^{1 / 2} .\right.
$$

Now, by using a Taylor's expansion

$$
\left(\lambda_{1 / 2}^{+}\right)_{-1}=-e^{i \pi / 4} \sqrt{-\tau}\left(1-\frac{i V(x)}{2 \tau}\right)
$$

we have the following estimates $\widetilde{L}_{\epsilon, 0}^{3}=\widetilde{L}_{\epsilon,-1 / 2}^{3}=\widetilde{L}_{\epsilon,-1}^{3}=1$ and

$$
\begin{aligned}
\widetilde{L}_{\epsilon, 1 / 2}^{3} & =\mid \exp \left(-2 e^{-i \pi / 4}\left(\epsilon \sqrt{-\tau}+\frac{i}{2 \sqrt{-\tau}} \int_{-\epsilon / 2}^{\epsilon / 2} V(y) d y\right) \mid\right. \\
& =\left|\exp \left(-\epsilon \sqrt{-2 \tau}-\frac{1}{\sqrt{-2 \tau}} \int_{-\epsilon / 2}^{\epsilon / 2} V(y) d y\right)\right|
\end{aligned}
$$

The proof follows from $\widetilde{L}_{\epsilon}^{C, 3}=\widetilde{L}_{\epsilon, 1 / 2}^{3}$.

More specific contraction factors can be established. For a small overlap $\epsilon$, we observe that we have the following estimates through the midpoint quadrature rule and a Taylor expansion with respect to $\epsilon$

$$
L_{\epsilon}^{C, 0}(\tau) \approx\left|\exp \left(-2 e^{-i \pi / 4} \epsilon \sqrt{-\tau+i V(0)}\right)\right|
$$

\subsection{Asymptotic estimates of the contraction factor in the OSWR algorithm}

We now study the rate of convergence of the Optimized Schwarz Waveform Relaxation (OSWR) method [31] as a function of the order of the transmitting boundary conditions which are used. If we assume that $\phi^{ \pm,(0)}(\mp \epsilon / 2, \cdot)$ and $\phi_{0}^{ \pm}$are some given functions, the OSWR algorithm at iteration $k \geq 1$ reads as follows

$$
\left\{\begin{array}{l}
P \phi^{ \pm,(k)}=0, \text { in } \Omega_{\epsilon}^{ \pm} \times \mathbb{R}_{+}^{*}, \\
\phi^{ \pm,(k)}(\cdot, 0)=\phi_{0}^{ \pm}, \text {in } \Omega_{\epsilon}^{ \pm} \\
\left(\partial_{x}+i \Lambda^{ \pm, p}\right) \cdot \phi^{ \pm,(k)}( \pm \epsilon / 2, \cdot)=\left(\partial_{x}+i \Lambda^{ \pm, p}\right) \cdot \phi^{\mp,(k-1)}( \pm \epsilon / 2, \cdot) \text { in } \mathbb{R}_{+}^{*}
\end{array}\right.
$$

where, in the same spirit as (35), we set: $\Lambda^{ \pm, p}=\operatorname{Op}\left(\lambda^{ \pm, p}\right)$, for $p=1 / 2,0,-1 / 2 \ldots$ Following [24], we formally define in $\Omega_{\epsilon}^{ \pm}$

$$
P \cdot e_{P}^{O, \pm, p}=0 \text { on } \Omega_{\epsilon}^{ \pm, p} \times \mathbb{R}_{+}^{*}, \quad\left(\partial_{x}+i \Lambda^{ \pm, p}\right) \cdot e_{P}^{O, \pm, p}( \pm \epsilon / 2, t)=h_{\epsilon}^{ \pm}(t) \text { on }\{ \pm \epsilon / 2\} \times \mathbb{R}_{+}^{*},
$$

and we introduce the mapping

$$
\mathcal{G}_{P}^{O, p}:\left\langle h_{\epsilon}^{+}, h_{\epsilon}^{-}\right\rangle \mapsto\left\langle\left(\partial_{x}+i \Lambda^{+, p}\right) \cdot e_{P}^{O,-, p}(\epsilon / 2, \cdot),\left(\partial_{x}+i \Lambda^{-, p}\right) \cdot e_{P}^{O,+, p}(-\epsilon / 2, \cdot)\right\rangle .
$$

The aim of the next result is to derive some asymptotic estimates of the convergence rate of the OSWR DDM when a transmitting boundary condition based on the symbol approximation involving $\lambda^{ \pm, p}$ is considered (system (49)). A corollary is also stated when the truncated symbolical 
expansion is Taylorized to get boundary operators based on $\tilde{\lambda}^{ \pm, p}$ (corollary 2.1). Let us introduce the following approximate boundary-value problem related to the OSWR method

$$
\left\{\begin{array}{l}
\left(\partial_{x}+i \Lambda^{\mp}\right) \cdot e_{\Lambda}^{O, \pm, p}=0, \text { in } \Omega_{\varepsilon}^{ \pm} \times \mathbb{R}_{+}^{*}, \\
\left(\partial_{x}+i \Lambda^{ \pm, p}\right) \cdot e_{\Lambda}^{O, \pm, p}( \pm \epsilon / 2, t)=h_{\epsilon}^{ \pm}(t), \text { on }\{ \pm \epsilon / 2\} \times \mathbb{R}_{+}^{*} .
\end{array}\right.
$$

Then, the following result holds.

Theorem 2.2. Let us assume that $V(x)$ is a smooth one-dimensional potential and that $\lambda^{ \pm}$is approximated by

$$
\lambda^{ \pm} \approx \lambda^{ \pm, p}:=\sum_{j=0}^{p} \lambda_{1 / 2-j / 2}^{ \pm},
$$

with $|\tau| \gg 1$ and $p \in \mathbb{N}^{*}$. An asymptotic estimate of the contraction factor $C_{P, \epsilon}^{O}$ of the mapping $\mathcal{G}_{P}^{O, p 2}$ (with $\mathcal{G}_{P}^{O, p 2}=\mathcal{G}_{P}^{O, p} \circ \mathcal{G}_{P}^{O, p}$ ), defined by (51), for the fixed-point OSWR algorithm (49) is given by

$$
C_{P, \epsilon}^{O} \approx C_{\epsilon}^{O, p}=\sup _{\tau \in \mathcal{H}_{\tau}} L_{\epsilon}^{O, p}(\tau)
$$

where

$$
L_{\epsilon}^{O, p}(\tau) \approx c_{\epsilon}^{p} \frac{1}{\left|\lambda_{1 / 2}^{+}(\epsilon / 2, \tau) \lambda_{1 / 2}^{+}(-\epsilon / 2, \tau)\right|^{p+1}} L_{\epsilon}^{C, p}(\tau)
$$

In the previous expression, $c_{\epsilon}^{p}$ is an $(\epsilon, p, V)$-dependent positive real-valued constant. The principal symbol is given by (10) and $L_{\epsilon}^{C, p}(\tau)$ designates the estimate of the convergence rate of the CSWR method given by equation (34).

For a constant potential $V$, the fixed point OSWR algorithm (49) converges in two iterations for the one-dimensional potential-free case, even without overlap $(\epsilon=0)$.

Proof. Let us introduce the approximate problem (52) which leads to the approximate representation $\hat{e}_{\Lambda}^{O, \pm, p}$ of the error $\hat{e}_{P}^{O, \pm, p}$. Like for the CSWR method, the strategy for the OSWR approach consists in evaluating the convergence rate of the operator (called later $\mathcal{G}_{\Lambda}^{O, p}$, see (56)) related to problem (52) to approximate the exact convergence rate of the application $\mathcal{G}_{P}^{O, p}$ given by (51) for the initial problem (50). At the symbol level, we have from (52)

$$
\left\{\begin{array}{l}
\left(\partial_{x}+i \lambda^{\mp}\right) \cdot \hat{e}_{\Lambda}^{O, \pm, p}=0, \text { in } \Omega_{\varepsilon}^{ \pm} \times \mathbb{R}_{+}^{*}, \\
\left(\partial_{x}+i \lambda^{ \pm, p}\right) \cdot \hat{e}_{\Lambda}^{O, \pm, p}( \pm \epsilon / 2, t)=\hat{h}_{\epsilon}^{ \pm}(t), \text { on }\{ \pm \epsilon / 2\} \times \mathbb{R}_{+}^{*},
\end{array}\right.
$$

leading to the expression

$$
\hat{e}_{\Lambda}^{O, \pm, p}(x, \tau)=\alpha_{\epsilon}^{ \pm, p}(\tau) \exp \left(-i \int_{ \pm \epsilon / 2}^{x} \lambda^{\mp}(y, \tau) d y\right),
$$

for some functions $\alpha_{\epsilon}^{ \pm, p}$. By using the transmitting boundary conditions (second equation of system (54)), we obtain

$$
\hat{e}_{\Lambda}^{O, \pm, p}(x, \tau)=\frac{\hat{h}_{\epsilon}^{ \pm}(\tau)}{i\left(\lambda^{ \pm, p}( \pm \epsilon / 2, \tau)-\lambda^{\mp}( \pm \epsilon / 2, \tau)\right)} \exp \left(-i \int_{ \pm \epsilon / 2}^{x} \lambda^{\mp}(y, \tau) d y\right) .
$$


Let us now consider the mapping

$$
\mathcal{G}_{\Lambda}^{O, p}:\left\langle h_{\epsilon}^{+}, h_{\epsilon}^{-}\right\rangle \mapsto\left\langle\left(\partial_{x}+i \Lambda^{+, p}\right) \cdot e_{\Lambda}^{O,-, p}(\epsilon / 2, \cdot),\left(\partial_{x}+i \Lambda^{-, p}\right) \cdot e_{\Lambda}^{O,+, p}(-\epsilon / 2, \cdot)\right\rangle .
$$

We can then write that

$$
\begin{aligned}
& \mathcal{F}_{t}\left\langle\mathcal{G}_{\Lambda}^{O, p}\left(h_{\epsilon}^{+}, h_{\epsilon}^{-}\right)\right\rangle=\left\langle\left(\partial_{x}+i \lambda^{+, p}\right) \hat{e}_{\Lambda}^{O,-, p}(\epsilon / 2, \cdot),\left(\left(\partial_{x}+i \lambda^{-, p}\right) \hat{e}_{\Lambda}^{O,+, p}(-\epsilon / 2, \cdot)\right\rangle\right. \\
& =i\left\langle\left(\lambda^{+, p}(\epsilon / 2, \tau)-\lambda^{+}(\epsilon / 2, \tau)\right) \hat{e}_{\Lambda}^{O,-, p}(\epsilon / 2, \cdot),\left(\lambda^{-, p}(-\epsilon / 2, \tau)-\lambda^{-}(-\epsilon / 2, \tau)\right) \hat{e}_{\Lambda}^{O,+, p}(-\epsilon / 2, \cdot)\right\rangle \\
& =i\left\langle\sum_{j=p+1}^{+\infty} \lambda_{1 / 2-j / 2}^{-}(\epsilon / 2, \tau) \hat{e}_{\Lambda}^{O,-, p}(\epsilon / 2, \cdot), \sum_{j=p+1}^{+\infty} \lambda_{1 / 2-j / 2}^{+}(-\epsilon / 2, \tau) \hat{e}_{\Lambda}^{O,+, p}(-\epsilon / 2, \cdot)\right\rangle .
\end{aligned}
$$

To get an explicit expression of $\mathcal{F}_{t}\left(\mathcal{G}_{\Lambda}^{O, p 2}\left\langle h_{\epsilon}^{+}, h_{\epsilon}^{-}\right\rangle\right)$, we iterate one more time by fixing the boundary data $h_{\epsilon}^{ \pm,(2)}$ to

$$
\hat{h}_{\epsilon}^{ \pm, p,(2)}(\tau)=i\left(\lambda^{\mp}( \pm \epsilon / 2, \tau)+\lambda^{ \pm, p}( \pm \epsilon / 2, \tau)\right) \hat{e}_{\Lambda}^{O, \mp, p}( \pm \epsilon / 2, \tau)
$$

and then

$$
\hat{e}_{\Lambda}^{O, \pm, p,(2)}(x, \tau)=\frac{\lambda^{\mp}( \pm \epsilon / 2, \tau)+\lambda^{ \pm, p}( \pm \epsilon / 2, \tau)}{\lambda^{ \pm, p}( \pm \epsilon / 2, \tau)-\lambda^{\mp}( \pm \epsilon / 2, \tau)} \hat{e}_{\Lambda}^{O, \mp, p}( \pm \epsilon / 2, \tau) \exp \left(-i \int_{ \pm \epsilon / 2}^{x} \lambda^{\mp}(y, \tau) d y\right) .
$$

By using (55), this leads to

$$
\begin{aligned}
\hat{e}_{\Lambda}^{O, \pm, p,(2)}(x, \tau)= & \frac{\lambda^{\mp}( \pm \epsilon / 2, \tau)+\lambda^{ \pm, p}( \pm \epsilon / 2, \tau)}{\lambda^{ \pm, p}( \pm \epsilon / 2, \tau)-\lambda^{\mp}( \pm \epsilon / 2, \tau)} \times \frac{\hat{h}_{\epsilon}^{\mp}(\tau)}{i\left(\lambda^{\mp, p}(\mp \epsilon / 2, \tau)-\lambda^{ \pm}(\mp \epsilon / 2, \tau)\right)} \\
& \times \exp \left(-i \int_{\mp \epsilon / 2}^{ \pm \epsilon / 2} \lambda^{ \pm}(y, \tau) d y\right) \exp \left(-i \int_{ \pm \epsilon / 2}^{x} \lambda^{\mp}(y, \tau) d y\right) .
\end{aligned}
$$

Now, we have

$$
\begin{aligned}
\mathcal{F}_{t}\left(\mathcal{G}_{\Lambda}^{O, p}\right. & \left.\circ \mathcal{G}_{\Lambda}^{O, p}\left\langle h_{\epsilon}^{+}, h_{\epsilon}^{-}\right\rangle\right)(\tau) \\
& =\left\langle\left(\partial_{x}+i \lambda^{+, p}\right) \hat{e}_{\Lambda}^{O,-, p,(2)}(\epsilon / 2, \cdot),\left(\partial_{x}+i \lambda^{-, p}\right) \hat{e}_{\Lambda}^{O,+, p,(2)}(-\epsilon / 2, \cdot)\right\rangle .
\end{aligned}
$$

We then need to evaluate $\left(\partial_{x}+i \lambda^{ \pm, p}\right) \hat{e}_{\Lambda}^{O, \mp, p,(2)}( \pm \epsilon / 2, \cdot)$ through

$$
\begin{aligned}
\left(\partial_{x}+\right. & \left.i \lambda^{ \pm, p}\right) \cdot \hat{e}_{\Lambda}^{O, \mp, p,(2)}( \pm \epsilon / 2, \cdot) \\
& =\frac{\lambda^{\mp}( \pm \epsilon / 2, \tau)+\lambda^{ \pm, p}( \pm \epsilon / 2, \tau)}{\lambda^{ \pm, p}( \pm \epsilon / 2, \tau)-\lambda^{\mp}( \pm \epsilon / 2, \tau)} \times \frac{\lambda^{\mp, p}(\mp \epsilon / 2, \tau)-\lambda^{\mp}(\mp \epsilon / 2, \tau)}{\lambda^{\mp, p}(\mp \epsilon / 2, \tau)-\lambda^{ \pm}(\mp \epsilon / 2, \tau)} \\
& \times \exp \left(-2 i \int_{\mp \epsilon / 2}^{ \pm \epsilon / 2} \lambda^{ \pm}(y, \tau) d y\right) \hat{h}_{\epsilon}^{ \pm}(\tau) .
\end{aligned}
$$

Since $\lambda^{-}=-\lambda^{+}$and $\lambda^{-, p}=-\lambda^{+, p}$, we can simplify the above expression as

$$
\begin{aligned}
\left(\partial_{x}+\right. & \left.i \lambda^{ \pm, p}\right) \cdot \hat{e}_{\Lambda}^{O, \mp, p,(2)}( \pm \epsilon / 2, \cdot) \\
& =\frac{\lambda^{+, p}( \pm \epsilon / 2, \tau)-\lambda^{+}( \pm \epsilon / 2, \tau)}{\lambda^{+, p}( \pm \epsilon / 2, \tau)+\lambda^{+}( \pm \epsilon / 2, \tau)} \times \frac{\lambda^{+}(\mp \epsilon / 2, \tau)-\lambda^{+, p}(\mp \epsilon / 2, \tau)}{\lambda^{+, p}(\mp \epsilon / 2, \tau)+\lambda^{+}(\mp \epsilon / 2, \tau)} \\
& \times \exp \left(-2 i \int_{-\epsilon / 2}^{\epsilon / 2} \lambda^{+}(y, \tau) d y\right) \hat{h}_{\epsilon}^{\mp}(\tau) .
\end{aligned}
$$


Based on (58) and by using (60), one deduces that

$$
\begin{aligned}
& \mathcal{F}_{t}\left(\mathcal{G}_{\Lambda}^{O, p} \circ \mathcal{G}_{\Lambda}^{O, p}\left\langle h_{\epsilon}^{+}, h_{\epsilon}^{-}\right\rangle\right)(\tau) \\
& =\frac{\left[\lambda^{+, p}(\epsilon / 2, \tau)-\lambda^{+}(\epsilon / 2, \tau)\right]\left[\lambda^{+}(-\epsilon / 2, \tau)-\lambda^{+, p}(-\epsilon / 2, \tau)\right]}{\left[\lambda^{+, p}(\epsilon / 2, \tau)+\lambda^{+}(\epsilon / 2, \tau)\right]\left[\lambda^{+}(-\epsilon / 2, \tau)+\lambda^{+, p}(-\epsilon / 2, \tau)\right]} \\
& \times \exp \left(-2 i \int_{-\epsilon / 2}^{\epsilon / 2} \lambda^{+}(y, \tau) d y\right)\left\langle\hat{h}_{\epsilon}^{+}, \hat{h}_{\epsilon}^{-}\right\rangle(\tau) .
\end{aligned}
$$

When $p=+\infty$, we first notice that $\lambda^{+, p}$ coincides with $\lambda^{+}$implying that the exact convergence occurs in 2 iterations since the numerator in (61) is null

$$
\mathcal{F}_{t}\left(\mathcal{G}_{\Lambda}^{O, p} \circ \mathcal{G}_{\Lambda}^{O, p}\left\langle h_{\epsilon}^{+}, h_{\epsilon}^{-}\right\rangle\right)(\tau)=0 \times\left\langle\hat{h}_{\epsilon}^{+}, \hat{h}_{\epsilon}^{+}\right\rangle(\tau)
$$

In the special case where $V$ is constant and consequently $e_{P}^{O, \pm}=e_{\Lambda}^{O, \pm}$, we also recover that the OSWR algorithm converges in 2 iterations [24]. However, in practice, $p$ is a finite number. From (61), we can deduce an approximate rate of convergence $C_{\epsilon}^{O, p}$ of the OSWR algorithm with exact convergence rate $C_{P, \epsilon}^{O}$. From Proposition 2.3, we know that we have the following asymptotic control (for large $|-\tau+V| \gg 1$ ) of the remaining term

$$
\begin{aligned}
& \lambda^{+}( \pm \epsilon / 2, \tau)-\lambda^{+, p}( \pm \epsilon / 2, \tau)=\sum_{j=p+1}^{+\infty} \lambda_{1 / 2-j / 2}^{+}( \pm \epsilon / 2, \tau) \\
& =\frac{1}{\left(\lambda_{1 / 2}^{+}( \pm \epsilon / 2, \tau)\right)^{p}} \sum_{j=p+1}^{+\infty} \sum_{\ell=0}^{L_{j+1}} \frac{\mathcal{F}_{\ell}^{V,-j / 2}}{\left(\lambda_{1 / 2}^{+}\right)^{\ell}}( \pm \epsilon / 2, \tau)=\mathcal{O}\left(\frac{1}{\left(\lambda_{1 / 2}^{+}( \pm \epsilon / 2, \tau)\right)^{p}}\right) .
\end{aligned}
$$

Furthermore, we have

$$
\lambda^{+}( \pm \epsilon / 2, \tau)+\lambda^{+, p}( \pm \epsilon / 2, \tau)=2 \lambda_{1 / 2}^{+}( \pm \epsilon / 2, \tau)+\mathcal{O}(1)
$$

Collecting the various estimates, we conclude that

$$
\begin{aligned}
& \mathcal{F}_{t}\left(\mathcal{G}_{\Lambda}^{O, p 2}\left\langle h_{\epsilon}^{+}, h_{\epsilon}^{-}\right\rangle\right)(\tau)= \\
& \mathcal{O}\left(\frac{1}{\left(\lambda_{1 / 2}^{+}(\epsilon / 2, \tau) \lambda_{1 / 2}^{+}(-\epsilon / 2, \tau)\right)^{p+1}}\right) \times \exp \left(-2 i \int_{-\epsilon / 2}^{\epsilon / 2} \lambda^{+}(y, \tau) d y\right)\left\langle h_{\epsilon}^{+}, h_{\epsilon}^{-}\right\rangle(\tau) .
\end{aligned}
$$

As a consequence and truncating the symbolical expansion within the exponential term, an estimate of the convergence rate of the OSWR DDM is given by

$$
C_{\epsilon}^{O, p}=\sup _{\tau \in \mathcal{H}_{\tau}} L_{\epsilon}^{O, p}(\tau)
$$

with

$$
L_{\epsilon}^{O, p}(\tau) \approx c_{\epsilon}^{p} \frac{1}{\left|\lambda_{1 / 2}^{+}(\epsilon / 2, \tau) \lambda_{1 / 2}^{+}(-\epsilon / 2, \tau)\right|^{p+1}} L_{\epsilon}^{C, p}(\tau)
$$

where $L_{\epsilon}^{C, p}(\tau)$ is given by $(34), p \in \mathbb{N}^{*}$, and $c_{\epsilon}^{p}$ is an $\epsilon$-, $p$ - and $V$-dependent positive real-valued constant. 
Let us consider some examples now. We proved in Theorem 2.1 that

$$
L_{\epsilon}^{C}(\tau) \approx L_{\epsilon}^{C, 3}(\tau):=\left|\left(\frac{\tau-i V(-\epsilon / 2)}{\tau-i V(+\epsilon / 2)}\right)^{1 / 2}\right|\left|\exp \left(-2 e^{-i \pi / 4} \int_{-\epsilon / 2}^{\epsilon / 2} \sqrt{-\tau+i V(y)} d y\right)\right| .
$$

Consequently, ones gets

$$
C_{\epsilon}^{O, 0}=c_{\varepsilon}^{0} \sup _{\tau \in \mathcal{H}_{\tau}}\left|\frac{1}{(\tau-i V(\epsilon / 2))^{1 / 2}(\tau-i V(-\epsilon / 2))^{1 / 2}}\right|\left|\exp \left(-2 e^{-i \pi / 4} \int_{-\epsilon / 2}^{\epsilon / 2} \sqrt{-\tau+i V(y)} d y\right)\right|
$$

and

$$
C_{\epsilon}^{O, 3}=c_{\varepsilon}^{3} \sup _{\tau \in \mathcal{H}_{\tau}}\left|\frac{1}{(\tau-i V(-\epsilon / 2))^{3 / 2}(\tau-i V(\epsilon / 2))^{5 / 2}}\right|\left|\exp \left(-2 e^{-i \pi / 4} \int_{-\epsilon / 2}^{\epsilon / 2} \sqrt{-\tau+i V(y)} d y\right)\right|
$$

for some positive constants $c_{\varepsilon}^{0}$ and $c_{\varepsilon}^{3}$. If we consider now a small overlapping region, then one has

$$
C_{\epsilon}^{O, 0} \approx c_{\varepsilon}^{0} \sup _{\tau \in \mathcal{H}_{\tau}}\left|\frac{1}{\tau-i V(0)}\right|\left|\exp \left(-2 e^{-i \pi / 4} \epsilon \sqrt{-\tau+i V(0)}\right)\right|
$$

and

$$
C_{\epsilon}^{O, 3} \approx c_{\varepsilon}^{3} \sup _{\tau \in \mathcal{H}_{\tau}}\left|\frac{1}{(\tau-i V(0))^{4}}\right|\left|\exp \left(-2 e^{-i \pi / 4} \epsilon \sqrt{-\tau+i V(0)}\right)\right|
$$

We remark that these asymptotic estimates show that the OSWR DDM converges (unlike the CSWR method) even with no overlap because of the remaining factor. In addition, at least for large frequencies, the convergence is improved with respect to the order $p$ of the approximation.

Now, in practice, the Taylorized version of the truncated symbolical expansion is rather used for transmitting conditions. Similarly to theorem 2.2, the following result holds.

Corollary 2.1. Let $V(x)$ be a regular one-dimensional potential. We assume that $\lambda^{ \pm}$is approximated by

$$
\lambda^{ \pm} \approx \tilde{\lambda}^{ \pm, p}:=\sum_{j=0}^{p}\left(\lambda_{1 / 2-j / 2}^{ \pm}\right)_{(1-p) / 2},
$$

for $|\tau| \gg 1$ and $p \in \mathbb{N}^{*}$. The contraction factor $C_{P, \epsilon}^{O}$ of the mapping $\mathcal{G}_{P}^{O, p 2}$ given by (51) for the fixed-point $O S W R$ algorithm (49) can be estimated by

$$
C_{P, \epsilon}^{O} \approx \tilde{C}_{\epsilon}^{O, p}=\sup _{\tau \in \mathcal{H}_{\tau}} \tilde{L}_{\epsilon}^{O, p}(\tau)
$$

where

$$
\tilde{L}_{\epsilon}^{O, p}(\tau) \approx \tilde{C}_{\epsilon}^{p} \frac{1}{|\tau|^{p+1}} \tilde{L}_{\epsilon}^{C, p}(\tau)
$$

The positive constant $\tilde{c}_{\epsilon}^{p}$ is $(\epsilon, p, V)$-dependent and $\tilde{L}_{\epsilon}^{C, p}(\tau)$ designates the convergence rate estimate of the CSWR DDM given by equation (42). 


\subsection{Convergence of the Classical and Optimized Schwarz Waveform Relaxation algorithms}

From the evaluation of the contraction factor and by using a similar analysis as in [24], we can deduce an asymptotic convergence result (Theorem 2.3). At any Schwarz iteration $k$, we denote by $T^{(k)}$ the convergence time of the CNGF algorithm thanks to the stopping criterion: $\phi(\cdot, t)=\phi\left(\cdot, T^{(k)}\right)$, for any $t \geq T^{(k)}$. In practice, we introduce a positive parameter $\delta$ and, at Schwarz iteration $k$, the imaginary-time iterations are stopped when, for $n \geq 0$, ones gets

$$
\left\|\phi^{ \pm,(k)}\left(\cdot, t_{n+1}^{-}\right)-\phi^{ \pm,(k)}\left(\cdot, T^{(k)}\right)\right\|_{L^{\infty}(\mathbb{R})} \leq \delta .
$$

To prove the result, we assume that the sequence of stopping times $\left\{T^{(k)}\right\}_{k}$ i) satisfies $T^{(k)} \leq T^{(k-1)}$ (at least for $k$ large enough) and ii) is convergent to $T^{\left(k^{\mathrm{cvg}}\right)}>0$. This last assumption is morally reasonable and will be confirmed numerically (see Section 3). It means that the larger the iteration $k$, the faster the CNGF algorithm to reach the stationary state. By extension of Theorem 5.8 in [24], we have

Theorem 2.3. Let us assume that i) $V$ is a smooth and bounded spatial dependent function, ii) the sequence $\left\{T^{(k)}\right\}_{k}$ is decreasing and convergent to $T^{(\infty)}>0$, i.e. there exists $k_{0}$ such that $0<T^{(\infty)} \leq T^{(k)} \leq T^{(k-1)}$ for all $k \geq k_{0}$, with $\lim _{k \rightarrow+\infty} T^{(k)}=T^{(\infty)}$, and iii) $T^{\left(k_{0}\right)}$ is finite. Then, the following inequalities hold

$$
\left\|e_{\Lambda}^{O, C, \pm}\right\|_{L^{2}\left(\mathbb{R}_{+} ; H^{2}\left(\Omega_{\epsilon}^{ \pm}\right)\right)} \leq C_{\Lambda, \epsilon}^{C, O}\left\|h_{\epsilon}^{ \pm}\right\|_{\left(H^{3 / 4}\left(\mathbb{R}_{+}\right)\right)^{2}}
$$

and

$$
\begin{gathered}
\left\|\left(\left(e_{\Lambda}^{O, C,+}\right)^{2 k+1},\left(e_{\Lambda}^{O, C,-}\right)^{2 k+1}\right)\right\|_{H^{3,3 / 2}\left(\Omega_{\epsilon}^{+} \times\left(0, T^{\left(k_{0}\right)}\right)\right) \times H^{3,3 / 2}\left(\Omega_{\epsilon}^{-} \times\left(0, T^{\left(k_{0}\right)}\right)\right)} \\
\leq D\left(C_{\Lambda, \epsilon}^{C, O}\right)^{k}\left\|\left(h_{\epsilon}^{+, 0}, h_{\epsilon}^{-, 0}\right)\right\|_{\left(H^{3 / 4}\left(0, T^{\left(k_{0}\right)}\right)\right)^{2}}
\end{gathered}
$$

where $D$ is a constant and the initial guess is null in $\Omega_{\epsilon}^{ \pm}$. The constant $C_{\Lambda, \epsilon}^{C, O}$ is defined as the contraction factor of the mapping $\mathcal{G}_{\Lambda}^{C, O, p 2}$.

To prove this theorem, we first start by stating the following Lemma.

Lemma 2.1. We denote by $P$ the linear imaginary-time Schrödinger operator, $\Omega$ an open subset of $\mathbb{R}$ and $\phi_{0}$ in $L^{2}(\Omega)$. Now, let $\widetilde{\phi}$ designates the solution to

$$
\left\{\begin{array}{l}
\partial_{t} \widetilde{\phi}=P \widetilde{\phi}, \text { on } \Omega \times \mathbb{R}_{+}^{*}, \\
\widetilde{\phi}(\cdot, 0)=\phi_{0}(\cdot), \text { on } \Omega,
\end{array}\right.
$$

and $\widetilde{\psi}$ the solution to

$$
\left\{\begin{array}{l}
\partial_{t} \widetilde{\psi}=P \widetilde{\psi}, \text { on } \Omega \times \mathbb{R}_{+}^{*} \\
\widetilde{\psi}(\cdot, 0)=\frac{\phi_{0}(\cdot)}{\left\|\phi_{0}(\cdot)\right\|_{L^{2}}}, \text { on } \Omega
\end{array}\right.
$$

Then, for any $T>0$, we have $\phi(\cdot, T)=\psi(\cdot, T)$, where

$$
\phi(\cdot, T):=\frac{\widetilde{\phi}(\cdot, T)}{\|\widetilde{\phi}(\cdot, T)\|_{L^{2}}}, \psi(\cdot, T):=\frac{\widetilde{\psi}(\cdot, T)}{\|\widetilde{\psi}(\cdot, T)\|_{L^{2}}} .
$$


Proof. The proof of Theorem 2.3 is a consequence of i) the analysis presented in [24], ii) Lemma 2.1 , iii) the decay of $T^{(k)}$ with respect to $k$ and convergent to $T^{(\infty)}>0$, and iv) that at any iteration $k \geq 2$

$$
\begin{aligned}
\left\|\mathcal{G}_{\Lambda}^{C, O} \circ \mathcal{G}_{\Lambda}^{C, O}\left(h_{\epsilon}^{+}, h_{\epsilon}^{+}\right)\right\|_{\left(H^{3 / 4}\left(0, T^{(k)}\right)\right)^{2}} & \leq C_{\Lambda, \epsilon}^{C, O}\left\|\left(h_{\epsilon}^{+}, h_{\epsilon}^{-}\right)\right\|_{\left(H^{3 / 4}\left(0, T^{(k)}\right)\right)^{2}} \\
& \leq C_{\Lambda, \epsilon}^{C, O}\left\|\left(h_{\epsilon}^{+}, h_{\epsilon}^{-}\right)\right\|_{\left(H^{3 / 4}\left(0, T^{(k-2)}\right)\right)^{2}},
\end{aligned}
$$

for any $h_{\epsilon}^{ \pm} \in H^{3 / 4}\left(0, T^{(k-2)}\right)$. This last statement is a consequence of Theorems 2.1 and 2.2 and is valid due to the linearity of the Schrödinger equation in imaginary-time. Indeed, the analysis provided in Sections 2.2 and 2.3 is performed on a fixed time interval $(0, T)$ and without normalization. In other words, at any Schwarz iteration $k$ and for any imaginary time interval $\left(t_{n}, t_{n+1}\right)$, $n \geq 0$, Theorems 2.1 and 2.2 hold. In the CNGF method, at any Schwarz iteration $k$ and at any time $t_{n+1}^{-} \leq T^{(k)}$, we normalize the computed solution (see system (3)). This process is performed at each time iteration until convergence at time $T^{(k)}$. For the linear operator $P$ and for a final and finite convergence time $T^{(k)}$, the normalization can be performed only once at time $T^{(k)}$ according to Lemma 2.1. In other words, for linear operators, the inequality (76) is valid with or without normalization at each time iteration. Now, at iteration $2 k$, since

$$
\left(h_{\epsilon}^{+,(2 k)}, h_{\epsilon}^{-,(2 k)}\right)=\mathcal{G}_{\Lambda}^{C, O} \circ \mathcal{G}_{\Lambda}^{C, O}\left(h_{\epsilon}^{+,(2 k-2)}, h_{\epsilon}^{-,(2 k-2)}\right),
$$

we can also write that

$$
\left\|\left(h_{\epsilon}^{+,(2 k)}, h_{\epsilon}^{-,(2 k)}\right)\right\|_{\left(H^{3 / 4}\left(0, T^{(2 k)}\right)\right)^{2}} \leq C_{\Lambda, \epsilon}^{C, O}\left\|\left(h_{\epsilon}^{+,(2 k-2)}, h_{\epsilon}^{-,(2 k-2)}\right)\right\|_{\left(H^{3 / 4}\left(0, T^{(2 k-2)}\right)\right)^{2}},
$$

where we have $h_{\epsilon}^{ \pm,(k)}=\mathcal{B}_{ \pm}^{C, O} \cdot e^{ \pm,(k)}( \pm \epsilon / 2, \cdot)$. In the previous formulae, we introduce i) $\mathcal{B}_{ \pm}^{C, O}$ as the

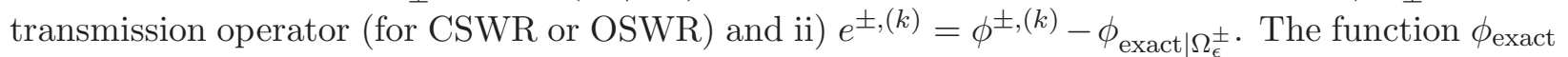
is the exact solution to (3). Now, since $\left(T^{(k)}\right)_{k}$ is decreasing for $k \geq k_{0}$ and converges to $T^{(\infty)}>0$, we can extend $h_{\epsilon}^{ \pm, 2 k}$ defined on $\left(0, T^{(2 k)}\right)$ by zero on $\left(T^{(2 k)}, T^{(2 k-2)}\right)$ in $H\left(0, T^{(2 k-2)}\right)$ and we next have

$$
\left\|\left(h_{\epsilon}^{+,(2 k)}, h_{\epsilon}^{-,(2 k)}\right)\right\|_{\left(H^{3 / 4}\left(0, T^{(2 k-2)}\right)\right)^{2}} \leq C_{\Lambda, \epsilon}\left\|\left(h_{\epsilon}^{+,(2 k-2)}, h_{\epsilon}^{-,(2 k-2)}\right)\right\|_{\left(H^{3 / 4}\left(0, T^{(2 k-2)}\right)\right)^{2}} .
$$

We then deduce by induction that

$$
\left\|\left(h_{\epsilon}^{+,(2 k)}, h_{\epsilon}^{-,(2 k)}\right)\right\|_{\left(H^{3 / 4}\left(0, T^{\left(k_{0}\right)}\right)\right)^{2}} \leq C C_{\Lambda, \epsilon}^{k}\left\|\left(h_{\epsilon}^{+,(0)}, h_{\epsilon}^{-,(0)}\right)\right\|_{\left(H^{3 / 4}\left(0, T^{\left(k_{0}\right)}\right)\right)^{2}} .
$$

The rest of the proof is similar to the one of Theorem 5.8 in [24].

For $k$ large enough or for $\phi_{0}$ sufficiently close to an eigenfunction (denoted by $\phi_{s}$ ), we expect that the results remain valid for the GPE. Indeed, in both cases, the function $\phi^{(k)}$ is close to an eigenstate and consequently the nonlinearity $\kappa\left|\phi^{(k)}(\cdot, t)\right|^{2}$ is supposed to behave almost like a fixed linear potential. In other words, from (40), we asymptotically guess that the contraction factor for the CSWR algorithm (denoted by $L_{\epsilon}^{\mathrm{GP}, C}$ ) behaves like

$$
\begin{aligned}
L_{\epsilon}^{\mathrm{GP}, C}(\tau) \approx & L_{\epsilon}^{\mathrm{GP}, C, 3}(\tau):=\left|\left(\frac{\tau-i V(-\epsilon / 2)-i \kappa\left|\phi_{s}(-\epsilon / 2)\right|^{2}}{\tau-i V(+\epsilon / 2)-i \kappa\left|\phi_{s}(\epsilon / 2)\right|^{2}}\right)^{1 / 2}\right| \\
& \times\left|\exp \left(-2 e^{-i \pi / 4} \int_{-\epsilon / 2}^{\epsilon / 2} \sqrt{-\tau+i V(y)+i \kappa\left|\phi_{s}(y)\right|^{2}} d y\right)\right|
\end{aligned}
$$


or, from (42), like

$$
L_{\epsilon}^{\mathrm{GP}, C}(\tau) \approx \widetilde{L}_{\epsilon}^{\mathrm{GP}, C, 3}(\tau):=\exp \left(-\epsilon \sqrt{-2 \tau}-\frac{1}{\sqrt{-2 \tau}} \int_{-\epsilon / 2}^{\epsilon / 2} V(y)+\kappa\left|\phi_{s}(y)\right|^{2} d y\right) .
$$

\section{Numerical validation of the theoretical asymptotic results}

\subsection{Discretization and algorithms}

Let us first describe how a consistent discretization is realized for the SIE scheme (4) applied to the imaginary-time version of the LSE/GPE based on the CNGF (3).

We consider the ABCs of order $p \in \mathbb{N}^{*}$ for the LSE

$$
\partial_{\mathbf{n}} \phi+i \widetilde{\Lambda}^{+, p}\left(x, t, \partial_{x}, \partial_{t}\right) \phi=0,
$$

with $\widetilde{\Lambda}^{+, p}=\operatorname{Op}\left(\widetilde{\lambda}^{+, p}\right)$. For the LSE, an equivalent form of the ABCs (77) can be obtained (see [6], Corollary 2, page 321) in imaginary-time as follows

$$
\left\{\begin{array}{l}
\widetilde{\Lambda}^{+, 1}\left(x, t, \partial_{x}, \partial_{t}\right) \phi=-i e^{i \Phi} \partial_{t}^{1 / 2}\left(e^{-i \Phi} \phi\right) \\
\widetilde{\Lambda}^{+, 4}\left(x, t, \partial_{x}, \partial_{t}\right) \phi=\widetilde{\Lambda}^{+, 1} \phi-\frac{i}{4} \partial_{\mathbf{n}}(V(x)) e^{i \Phi} I_{t}\left(e^{-i \Phi} \phi\right)
\end{array}\right.
$$

where we keep the same notations, in particular $\Phi(x, t)=t V(x)$. These forms of the boundary conditions are more adapted to the numerical treatment presented here. The formal extension to the nonlinear case [5] for the GPE is based on the formal operation $V(x) \rightarrow V(x)+\kappa|\phi(x, t)|^{2}$ and yields

$$
\left\{\begin{array}{l}
\widetilde{\Lambda}^{+, 1}\left(x, t, \partial_{x}, \partial_{t},|\phi|\right) \phi=-i e^{i \Phi} \partial_{t}^{1 / 2}\left(e^{-i \Phi} \phi\right), \\
\widetilde{\Lambda}^{+, 4}\left(x, t, \partial_{x}, \partial_{t},|\phi|\right) \phi=\widetilde{\Lambda}^{+, 1} \phi-\frac{i}{4} \partial_{\mathbf{n}}\left(\kappa|\phi|^{2}+V(x)\right) e^{i \Phi} I_{t}\left(e^{-i \Phi} \phi\right),
\end{array}\right.
$$

where the function $\Phi$ is defined for both the linear and nonlinear cases by

$$
\Phi(x, t)=t V(x)+\kappa \int_{0}^{t}|\phi(x, s)|^{2} d s .
$$

In the sequel of the paper, we designate by condition of order zero the first-order condition based on $\widetilde{\Lambda}^{+, 1}$ by forcing $V=0$ and $\kappa=0$. The discretizations of the nonlocal time operators are chosen as follows.

$$
\begin{gathered}
\partial_{t}^{1 / 2} f\left(t_{n}\right) \approx \sqrt{\frac{2}{\Delta t}} \sum_{k=0}^{n} \beta_{n-k} f^{k}, \\
I_{t} f\left(t_{n}\right) \approx \Delta t \sum_{k=1}^{n} f^{k},
\end{gathered}
$$

where the sequence $\left(\beta_{n}\right)_{n \in \mathbb{N}}$ is such that $\beta_{0}=1$ and, for $n \geq 0$,

$$
\beta_{n+1}=(-1)^{n} \frac{1-2 n}{2 n+2} \beta_{n} .
$$


The discretizations (80)-(81) are designed to be consistent with the implicit Euler scheme by using the associated generating function [2]. For (81), we use the right rectangle quadrature rule.

Let us recall that the problem is a priori set in an unbounded domain. However, we assume here that the solution $\phi$ remains confined within a finite computational domain $(-a, a)$, with $a>0$ large enough. This is a standard assumption in the framework of the numerical solution of the GPE. As a consequence, we can set a homogeneous Dirichlet boundary condition at $x= \pm a: \phi( \pm a, t)=0$. Let us remark that other boundary conditions could also be considered (see e.g. [12]). For the one-dimensional DDM, the subdomains of interest are then $\Omega_{a, \epsilon}^{+}=(-a, \epsilon / 2), \Omega_{a, \epsilon}^{-}=(-\epsilon / 2, a)$ and $\Omega_{a}=\Omega_{\epsilon}^{+} \cup \Omega_{\epsilon}^{-}=(-a, a)$, with the overlapping region $\Gamma_{\epsilon}=\Omega_{\epsilon}^{+} \cap \Omega_{\epsilon}^{-}=(-\epsilon / 2, \epsilon / 2)$, where $\epsilon \geq 0$. The solution $\phi^{+}$(respectively $\phi^{-}$) in $\Omega_{a, \epsilon}^{+}$(respectively $\Omega_{a, \epsilon}^{-}$) at time $t_{n+1}$ and Schwarz iteration $k$ (the time is also written $t_{n}^{(k)}$ later for some given $(n, k)$ when necessary) is denoted by $\phi^{+, n+1,(k)}$ (respectively $\phi^{-, n+1,(k)}$ ). The index $\pm \epsilon / 2$ designates the value of a function at point $x= \pm \epsilon / 2$ (e.g. $\left.\phi_{ \pm \epsilon / 2}=\phi( \pm \epsilon / 2)\right)$.

We consider a normalized initial guess $\phi_{0}$ and set $\left(\widetilde{\phi}^{+, 0,(k)}, \widetilde{\phi}^{-, 0,(k)}\right):=\left(\phi_{0}^{+}, \phi_{0}^{-}\right):=\left(\phi_{0 \mid \Omega_{a, \epsilon}^{+},}, \phi_{0 \mid \Omega_{a, \epsilon}^{-}}\right)$, for any $k \geq 0$. The semi-discrete OSWR-SIE scheme for a two-domains decomposition of the CNGF with a first-order ABC writes as follows

$$
\left\{\begin{array}{l}
\left(\frac{I}{\Delta t}-\partial_{x}^{2}+V(x)+\kappa\left|\phi^{ \pm, n,(k)}\right|^{2}\right) \widetilde{\phi}^{ \pm, n+1,(k)}=\frac{\phi^{ \pm, n,(k)}}{\Delta t}, \text { in } \Omega_{a, \epsilon}^{ \pm}, \\
\left(\partial_{\mathbf{n}^{ \pm}}+\sqrt{\frac{2}{\Delta t}}\right) \widetilde{\phi}_{ \pm \epsilon / 2}^{ \pm, n+1,(k)}=g_{ \pm \epsilon / 2}^{\mp, n+1,(k-1)}+\alpha_{ \pm \epsilon / 2}^{ \pm, n,(k)}-\alpha_{ \pm \epsilon / 2}^{\mp, n,(k-1)} \\
\widetilde{\phi}^{ \pm, n+1,(k)}=0, \text { at } x=\mp a .
\end{array}\right.
$$

At each iteration $(n+1, k)$, the global solution $\widetilde{\phi}^{n+1,(k)}$ needs to be normalized

$$
\phi^{n+1,(k)}:=\frac{\widetilde{\phi}^{+, n+1,(k)}+\widetilde{\phi}^{-, n+1,(k)}}{\left\|\widetilde{\phi}^{+, n+1,(k)}+\widetilde{\phi}^{-, n+1,(k)}\right\|_{L^{2}((-a, a))}} .
$$

The outwardly directed unit normal vector to $\Omega_{a, \epsilon}^{ \pm}$is denoted by $\mathbf{n}^{ \pm}$. We also set

$$
\begin{aligned}
& g_{ \pm \epsilon / 2}^{\mp, n+1,(k-1)}=\partial_{\mathbf{n}^{ \pm}} \widetilde{\phi}_{ \pm \epsilon / 2}^{\mp, n+1,(k-1)}+\sqrt{\frac{2}{\Delta t}} \widetilde{\phi}_{ \pm \epsilon / 2}^{\mp, n+1,(k-1)}, \\
& \alpha_{ \pm \epsilon / 2}^{\mp, n,(k)}=-\sqrt{\frac{2}{\Delta t}} E_{ \pm \epsilon / 2}^{\mp, n,(k)} \sum_{\ell=0}^{n} \beta_{n+1-\ell} \bar{E}_{ \pm \epsilon, 2}^{\mp, \ell,(k)} \widetilde{\phi}_{ \pm \epsilon / 2}^{\mp, \ell,(k)} \\
& E_{ \pm \epsilon / 2}^{\mp, n,(k)}=\exp \left(-\Delta t \sum_{q=0}^{n}\left(V_{ \pm \epsilon / 2}+\kappa\left|\phi_{ \pm \epsilon / 2}^{\mp, q,(k)}\right|^{2}\right)\right), \\
& \bar{E}_{ \pm \epsilon / 2}^{\mp, n,(k)}=\frac{1}{E_{ \pm \epsilon / 2}^{\mp, n,(k)}} .
\end{aligned}
$$

We use the following convergence criterion of the CNGF at Schwarz iteration $k$

$$
\left\|\phi^{n+1,(k)}-\phi^{n,(k)}\right\|_{\infty} \leq \delta
$$

with $\delta=10^{-8}$ and where $\|\psi\|_{\infty}:=\sup _{x \in \Omega_{a}}|\psi(x)|$. When the convergence is reached, the stopping time is: $T^{(k)}:=T^{\mathrm{cvg},(k)}=n^{\mathrm{cvg},(k)} \Delta t$ for a converged solution $\phi^{\mathrm{cvg},(k)}$ reconstructed from the two 
subdomains solutions $\phi^{ \pm, \mathrm{cvg},(k)}$. The convergence criterion for the Schwarz DDM is given by

$$
\|\| \phi_{\mid \Gamma_{\epsilon}}^{+, \operatorname{cvg},(k)}-\phi_{\mid \Gamma_{\epsilon}}^{-, \operatorname{cvg},(k)}\left\|_{\infty, \Gamma_{\epsilon}}\right\|_{L^{2}\left(0, T^{\left(k^{\mathrm{cvg}}\right)}\right)} \leq \delta^{\mathrm{Sc}},
$$

with $\delta^{\text {Sc }}=10^{-14}$ ("Sc" for Schwarz). When the convergence of the full iterative algorithm is obtained at Schwarz iteration $k^{\mathrm{cvg}}$, one gets the converged global solution $\phi^{\mathrm{cvg}}:=\phi^{\mathrm{cvg},\left(k^{\mathrm{cvg}}\right)}$ in $\Omega_{a}$. Let us remark that (82) is simple to implement since it is a standard CNGF-SIE scheme in each subdomain with homogeneous Dirichlet boundary conditions at the endpoint of the exterior domain and a local Fourier-Robin boundary condition at the transmitting endpoint. The extensions to the zeroth- and fourth-order boundary conditions are quite straightforward. We consider a spatial uniform grid and the Laplace operator is discretized by using a 3-points stencil.

\subsection{Numerical results in the linear case $(\kappa=0)$}

This subsection is devoted to some numerical experiments in the linear case. The computational domain $\Omega_{8}=(-8,8)$ is divided into two subdomains: $\Omega_{8, \epsilon}^{+}=(-8, \Delta x / 2), \Omega_{8, \epsilon}^{-}=(\Delta x / 2,8)$. The left and right subdomains are uniformly subdivided by using $N_{\epsilon}^{ \pm}=65$ points, including the two endpoints. The overlapping region is reduced to one cell of size $\Delta x=16 / 127$.

\subsubsection{Test 1.a}

In this first series of experiments, we consider the quadratic potential $V(x)=x^{2} / 2$. The initial data is $e^{-x^{2} / 2} \pi^{-1 / 4}$ and the imaginary time step is $\Delta t=2 \times 10^{-1}$.

In Fig. 1 (left), we report the convergence time of the CNGF, i.e. $T^{(k)}$, versus the Schwarz iterations $k$ for the CSWR DDM. The total number of iterations to get the convergence of the CSWR at machine tolerance is $k^{\mathrm{cvg}}=158$. We observe the decay of the sequence $\left(T^{(k)}\right)_{0 \leq k \leq k^{\mathrm{cvg}}}$ which is in accordance with the assumption in Theorem 2.3. In addition, we numerically obtain that $T^{\left(k^{\mathrm{cvg}}\right)} \approx 7.6$. Figure 1 (right) presents the energy $E_{0}^{\mathrm{cvg},(k)}$ of the converged solution $\phi^{\mathrm{cvg},(k)}$ computed at $T^{(k)}$ in $\Omega_{8}$ and defined by

$$
E_{\kappa}^{\mathrm{cvg},(k)}:=E_{\kappa}\left(\phi^{\mathrm{cvg},(k)}\right)=\int_{\Omega_{a}}\left|\nabla \phi^{\mathrm{cvg},(k)}\right|^{2}+V(\mathbf{x})\left|\phi^{\mathrm{cvg},(k)}\right|^{2}+\frac{\kappa}{2}|\chi(\mathbf{x})|^{4} d \mathbf{x} .
$$

Similarly to the standard global CNGF-SIE scheme, we observe that the energy is diminishing.
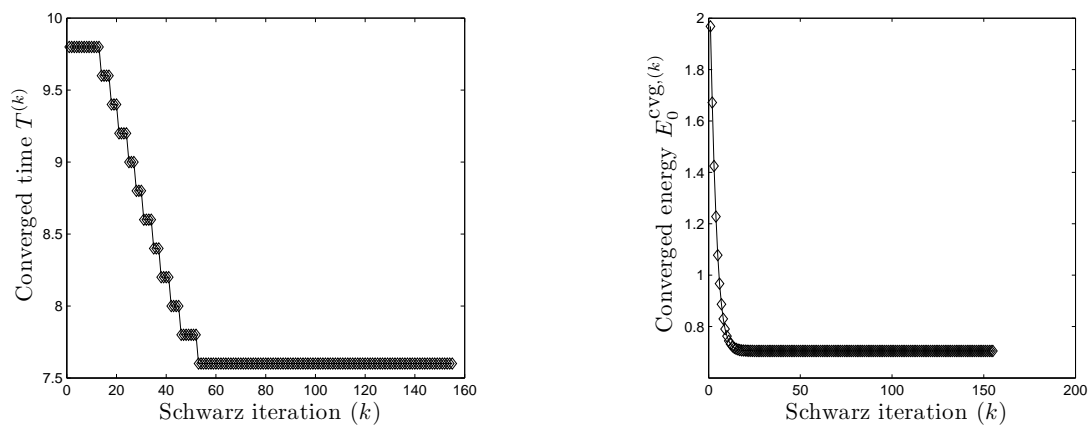

Figure 1: CSWR: stopping times $T^{(k)}$ (left) and total energy $E_{0}^{\mathrm{cvg},(k)}$ of the reconstructed CNGF converged solution $\phi^{\mathrm{cvg},(k)}$ (right) vs. iteration $k$ until convergence. 
Figure 2 (left) compares the numerical convergence rate obtained with the CNGF-SIE algorithm and the theoretical convergence rates (40) and (42) but written at the discrete level, i.e. we represent the $L^{2}$-norm error in time in the overlap (85). Then, we have $\epsilon=\Delta x$ and the largest time frequency is given by $1 / \Delta t$. According to (40), this leads to

$$
\begin{aligned}
& L_{\Delta x}^{C}(\tau) \approx \\
& \left|\left(\frac{1 / \Delta t-i V(-\Delta x / 2)}{1 / \Delta t-i V(+\Delta x / 2)}\right)^{1 / 2}\right|\left|\exp \left(-2 e^{-i \pi / 4} \int_{-\Delta x / 2}^{\Delta x / 2} \sqrt{-1 / \Delta t+i V(y)} d y\right)\right|
\end{aligned}
$$

which can be further approximated by a Taylor's expansion following (42) as

$$
L_{\Delta x}^{C}(\tau) \approx \exp \left(-\Delta x \sqrt{\frac{2}{\Delta t}}-V(0) \Delta x \sqrt{\frac{\Delta t}{2}}\right) .
$$

We observe an excellent agreement between the theoretical estimates and the computed convergence rates. Let us note that the estimate (87) based on the Taylor formula gives a contraction factor equal to 0.671379 when the nonlocal one (88) yields 0.671395 . We also report in Fig. 2 (right) the total energy $E_{0}^{n,(k)}:=E_{0}\left(\phi^{n,(k)}\right)$ of the reconstructed solution $\phi^{n,(k)}$ as a function of the imaginary time $t_{n}^{(k)}$ for different Schwarz iterations $k$. Let us note that although the SIE scheme with Dirichlet boundary condition is energy diminishing [16] in each subdomain, this property is not necessarily satisfied in the CSWR framework at time $t_{n}^{(k)}$ due to the arbitrary reconstruction (83) in the overlapping region $\Gamma_{\epsilon}$. However, if $k$ is large enough, the total energy is diminishing like for the global CNGF-SIE scheme.
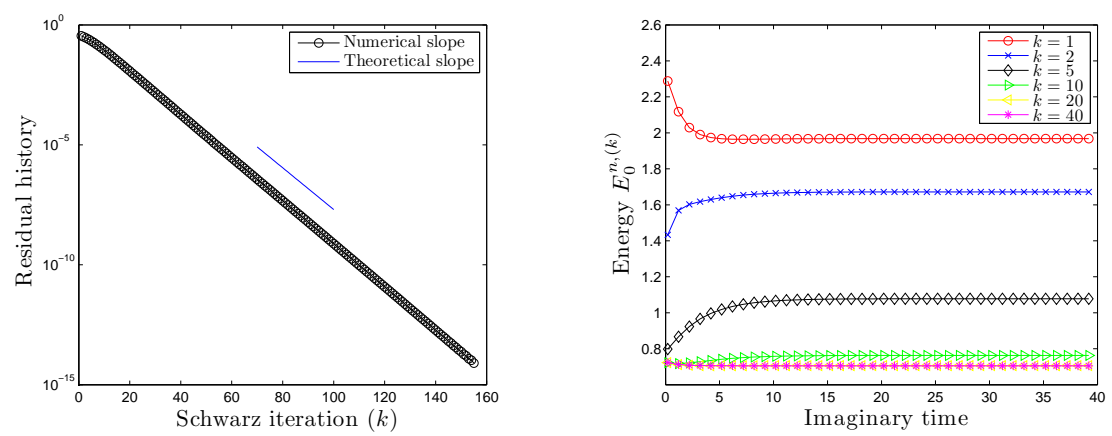

Figure 2: CSWR: left: comparison between the discrete versions of the estimated theoretical convergence rates (87) and (88) and the numerical ones. Right: total energy $E_{0}^{n,(k)}$ of the reconstructed solution $\phi^{n,(k)}$ for a few iterations $k$ vs. imaginary-time $t_{n}^{(k)}$.

Within the same framework as above, we compare in Figures 3 and 4 the convergence time and energy per Schwarz iteration $k$ (CNGF at convergence) as well as the CSWR convergence rate for various time steps $\Delta t$. On Figure 3 (left), we see that the convergence time for large values of $\Delta t(=1,5)$ decays from the beginning but can also exhibit an increase when the algorithm starts and then decays for smaller $\Delta t\left(=10^{-1}, 10^{-2}\right)$. This is again in accordance with the theoretical assumption given in Section 2.4. In addition, the number of Schwarz iterations is diminishing with respect to $\Delta t$, which was expected since it corresponds to increase the time frequency in formulae $(87)$ and (88). Figure 3 (right) reports the energy $E_{0}^{\mathrm{cvg},(k)}$ at convergence for each Schwarz iteration 
$k$. We see that it is decaying whatever $\Delta t$ is. For small $\Delta t$, the energy is decaying but presents some regions with extremely slow decay and then an instantaneous decay between two successive iterations to start a new regime with slowly decaying energy. Finally, Figure 4 shows the residual history of the error (85) vs. $k$, for various values of $\Delta t$. We observe that the slopes of the errors decay according to $\Delta t$.
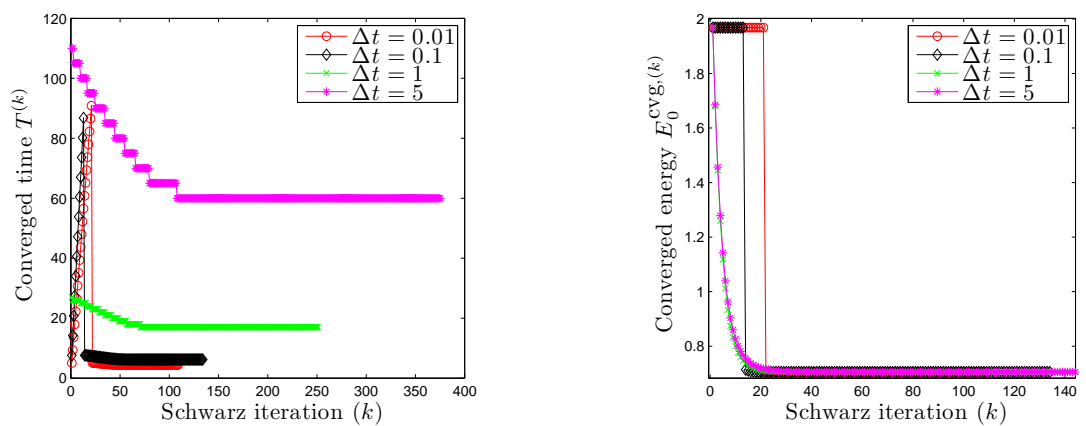

Figure 3: CSWR: stopping times $T^{(k)}$ (left) and total energy $E_{0}^{\mathrm{cvg},(k)}$ of the reconstructed CNGF converged solution $\phi^{\mathrm{cvg},(k)}$ (right) vs. iteration $k$ until convergence for various imaginary time steps $\Delta t$.

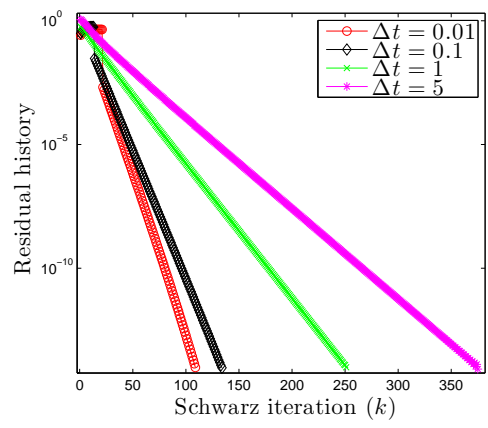

Figure 4: CSWR : residual history for various imaginary time steps $\Delta t$.

With the same data, we verify that the first-order OSWR contraction factor $\left|L_{\epsilon}^{0}(\tau)\right|$ behaves as $c_{\epsilon}\left|L_{\epsilon}^{C}(\tau) / \tau\right|$ (see Theorem 2.2) for an unknown time independent constant $c_{\epsilon}$, with $\epsilon=\Delta x$. More specifically, the approximate contraction factor at $x \approx 0$ for the OSWR method with first-order transmitting boundary conditions is

$$
L_{\Delta x}^{O}(\tau) \approx c_{\Delta x} \frac{1}{\left|\lambda_{1 / 2}^{+}(\Delta x / 2,1 / \Delta t) \lambda_{1 / 2}^{+}(-\Delta x / 2,1 / \Delta t)\right|} L_{\Delta x}^{C}(1 / \Delta t),
$$

or, by using a Taylor's expansion,

$$
\begin{aligned}
L_{\Delta x}^{O}(\tau) \approx & c_{\Delta x} \frac{1}{\sqrt{1 / \Delta t+i V(-\Delta x / 2)} \sqrt{1 / \Delta t+i V(\Delta x / 2)}} \\
& \times \exp \left(-\Delta x \sqrt{2 / \Delta t}-\Delta x V_{c}(0) \sqrt{\Delta t / 2}\right) .
\end{aligned}
$$


For a fixed overlapping region (reduced to one mesh element of size $\Delta x$ ), we compare the rate of convergence of the CSWR and first-order OSWR methods, but for different values of $\Delta t$, the latter being assumed to be small enough. Table 1 reports the theoretical (Theo) and numerical (Num) convergence rates of the CSWR and OSWR DDMs. In the last column, we report the estimated values of $c_{\Delta x}$ according to (89) for different time steps. As expected, we find $c_{\Delta x}$ as an almost time independent parameter, which a posteriori validates (89). We remark the excellent agreement between the theoretical and numerical CSWR-CNGF convergence rates.

Table 1: Comparison between the numerical and theoretical slopes of the CSWR and first-order OSWR DDMs and estimated value of $c_{\epsilon}$.

\begin{tabular}{cccccc}
\hline \hline$\Delta t$ & Num rate CSWR & Theo rate CSWR & Num rate OSWR & Theo rate OSWR & Estim $c_{\Delta x}$ \\
\hline $2.5 \times 10^{-2}$ & 0.330 & 0.324 & 0.001 & $0.008 c_{\Delta x}$ & 0.13 \\
$5.0 \times 10^{-2}$ & 0.481 & 0.451 & 0.004 & $0.023 c_{\Delta x}$ & 0.17 \\
$1.0 \times 10^{-1}$ & 0.566 & 0.569 & 0.094 & $0.057 c_{\Delta x}$ & 0.17 \\
$2.0 \times 10^{-1}$ & 0.653 & 0.671 & 0.19 & $0.134 c_{\Delta x}$ & 0.15 \\
\hline \hline
\end{tabular}

\subsubsection{Test 1.6}

We emphasize now on the effect of i) the interface location and ii) the order (of the transmitting boundary condition) of the OSWR algorithm on the convergence rate. We choose $V(x)=$ $5 \exp \left(-\alpha x^{2}\right)$ (which is non-zero in the overlapping region and is rapidly decaying) and $\Delta t=0.2$. For $\alpha=1$, we compare in Fig. 5 (left) the convergence rate of the CSWR algorithm for different interface locations: $x \approx 0,0.6,1$ (where $V \approx 5,2.6,0.8$ ), illustrating again the importance of the interface location to optimize the convergence rate. For $\alpha=2$, we also compare on Fig. 5 (right) the convergence rates of the CSWR and OSWR (orders 0,1 and 4) methods. The OSWR DDM of order 0 refers to as the potential-free $(V=0)$ Dirichlet-to-Neumann transmission condition. The choice $\alpha=2$ is justified by the need to have non negligible values of $V^{\prime}( \pm \Delta x / 2)$ in the overlapping region to justify the use of a fourth-order transmission condition. As expected, the higher the order, the faster the convergence.
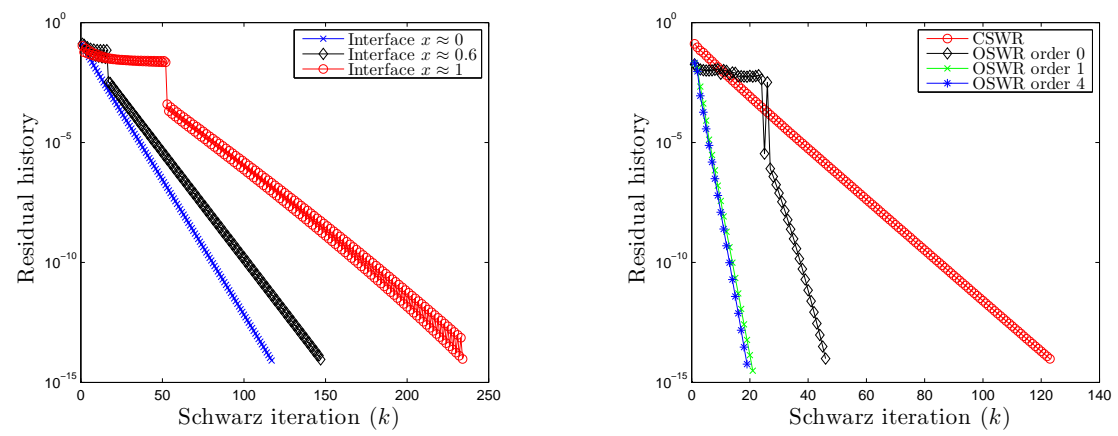

Figure 5: Left: CSWR DDM residual history for various interface locations $(\alpha=1)$. Right: residual history of the CSWR and OSWR DDMs $(\alpha=2)$.

\subsection{Numerical results in the nonlinear case $(\kappa \neq 0)$}

We consider now the imaginary-time GPE. Even if the analytical results presented in Section 2 were proven for the LSE, they should remain asymptotically valid for the GPE as discussed after 
Theorem 2.3.

\subsubsection{Test 2}

We consider the harmonic potential $V(x)=x^{2} / 2$ and the nonlinearity strength $\kappa=250$. The computational domains are $\Omega_{16}=(-16.16), \Omega_{16, \epsilon}^{+}=(-16, \Delta x / 2)$ and $\Omega_{16, \epsilon}^{-}=(-\Delta x / 2,16)$. We fix $\Delta t=1 \times 10^{-1}, N=512, N_{\epsilon}^{ \pm}=257$ and $\epsilon=\Delta x=32 / 511$. The initial guess is $x \mapsto e^{-x^{2} / 2} \pi^{-1 / 4}$ for which convergence to the ground state of the GPE is expected.

We report in Fig. 6 the converged solution (which is indeed the ground state) (left) as well as the convergence rate of the CSWR algorithm (right). In particular, we compare the numerical rate of convergence with the expected theoretical one

$$
\begin{aligned}
L_{\Delta x}^{\mathrm{GP}}(\tau) \approx \mid & \left(\frac{\Delta t-i V(-\Delta x / 2)-i \kappa\left|\phi_{s}(-\Delta x / 2)\right|^{2}}{\Delta t-i V(+\Delta x / 2)-i \kappa\left|\phi_{s}(\Delta x / 2)\right|^{2}}\right)^{1 / 2} \mid \\
& \times\left|\exp \left(-2 e^{-i \pi / 4} \int_{-\Delta x / 2}^{\Delta x / 2} \sqrt{-\Delta t+i V(y)+i \kappa\left|\phi_{s}(y)\right|^{2}} d y\right)\right|
\end{aligned}
$$

where $\tau$ is given by $1 / \Delta t$ leading to

$$
L_{\Delta x}^{\mathrm{GP}}(\tau) \approx \exp \left(-\Delta x \sqrt{2 / \Delta t}-\Delta x \sqrt{\Delta t / 2}\left(V(0)+\kappa\left|\phi_{\ell}(0)\right|^{2}\right)\right) .
$$

The computed numerical contraction factor is equal to 0.55. According to (90), we theoretically get the value 0.53 .
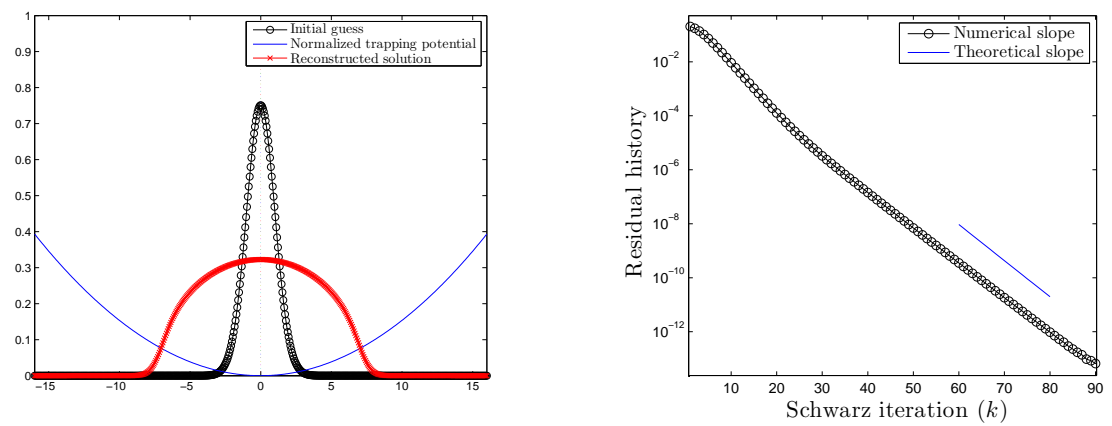

Figure 6: CSWR: left: converged solution (ground state). Right: comparison between the discrete versions of the estimated theoretical convergence rates (90) and the numerical ones for the CSWR DDM.

In Fig. 7, we report the results for the first excited state initializing now with $x \mapsto \sqrt{2} x e^{-x^{2} / 2} \pi^{-1 / 4}$. Numerically, the CSWR contraction factor is found to be 0.74 that should be compared with the theoretical value 0.75 obtained from (90). In both cases, we find a good agreement between the numerical and the theoretical values, validating the extension of the previous analysis for the LSE to the GPE.

\subsubsection{Test 3}

The last example consists in an optical potential $V(x)=x^{2} / 2+25 \cos ^{2}(\pi x / 2)$ and the nonlinear strength $\kappa=10$. We are interested in the convergence rate of both the CSWR and OSWR 

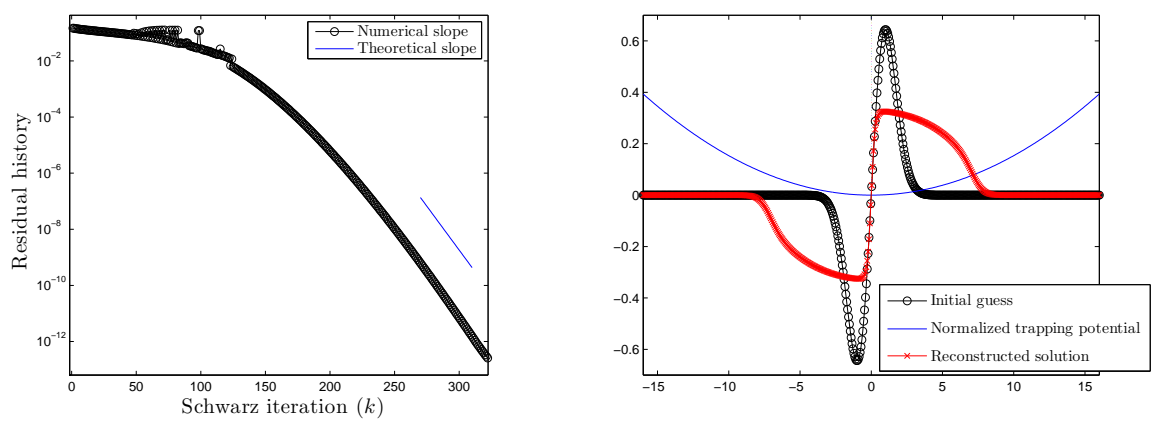

Figure 7: CSWR: left: converged solution (first excited state). Right: comparison between the discrete versions of the estimated theoretical convergence rates (90) and numerical ones for the CSWR DDM.

algorithms. We choose $\Omega_{8}=(-8,8), \Omega_{8, \epsilon}^{+}=(-8, \Delta x / 2)$ and $\Omega_{8, \epsilon}^{-}=(-\Delta x / 2,8)$. The overlapping region is again reduced to one element of size $\epsilon=\Delta x=16 / 255(N=256)$ and $N_{\epsilon}^{ \pm}=129$. In Fig. 8, we report the rates of convergence of the CSWR and the OSWR (orders 0 and 1) DDMs. If $\phi_{g}$ is the ground state of the GPE, let us remark that $\partial_{x}\left(V(x)+\kappa\left|\phi_{g}\right|^{2}(x)\right) \approx 0$ in the overlapping region. According to (79), it is then useless to perform a fourth-order OSWR method. For completeness, we also report the CNGF convergence time and energy per Schwarz iteration $k$ as well as the corresponding stationary state which are both uniformly decaying for the CSWR and the first-order OSWR method, but not for the zeroth-order OSWR algorithm.

\section{Conclusion}

This paper concerned the analysis of classical (CSWR) and optimized (OSWR) Schwarz waveform relaxation domain decomposition methods for computing in parallel the discrete spectrum of linear and nonlinear Schrödinger operators based on the Continuous Normalized Gradient Flow method. Using pseudodifferential calculus and the theory of artificial boundary conditions for LSEs, we analytically determined the rate of convergence of the CSWR and OSWR algorithms with respect to the order of approximation of the ABCs at the fictitious interfaces. We proved that not only the OSWR algorithm leads to the convergence of the non-overlapping DDM but that it also accelerates the CSWR method by a power of the time frequency in the high-frequency regime. Although the analysis was developed in the linear case with non-constant coefficients, the extension to the nonlinear Schrödinger operators (e.g. the GPE) was conjectured and numerically validated. It is important to point out that the mathematical methodology based on the theory of pseudodifferential operators that we developed here can be extended naturally to higher dimensional problems as well as other PDEs at the price of technical efforts. Several extensions of this paper are currently studied and are the topics of a forthcoming paper, in particular concerning the two-dimensional situation and the case of the real-time dynamics of LSEs/GPEs. Finally, the numerical analysis including the stability and convergence of the CNGF/SWR schemes will be developed in a future work. 

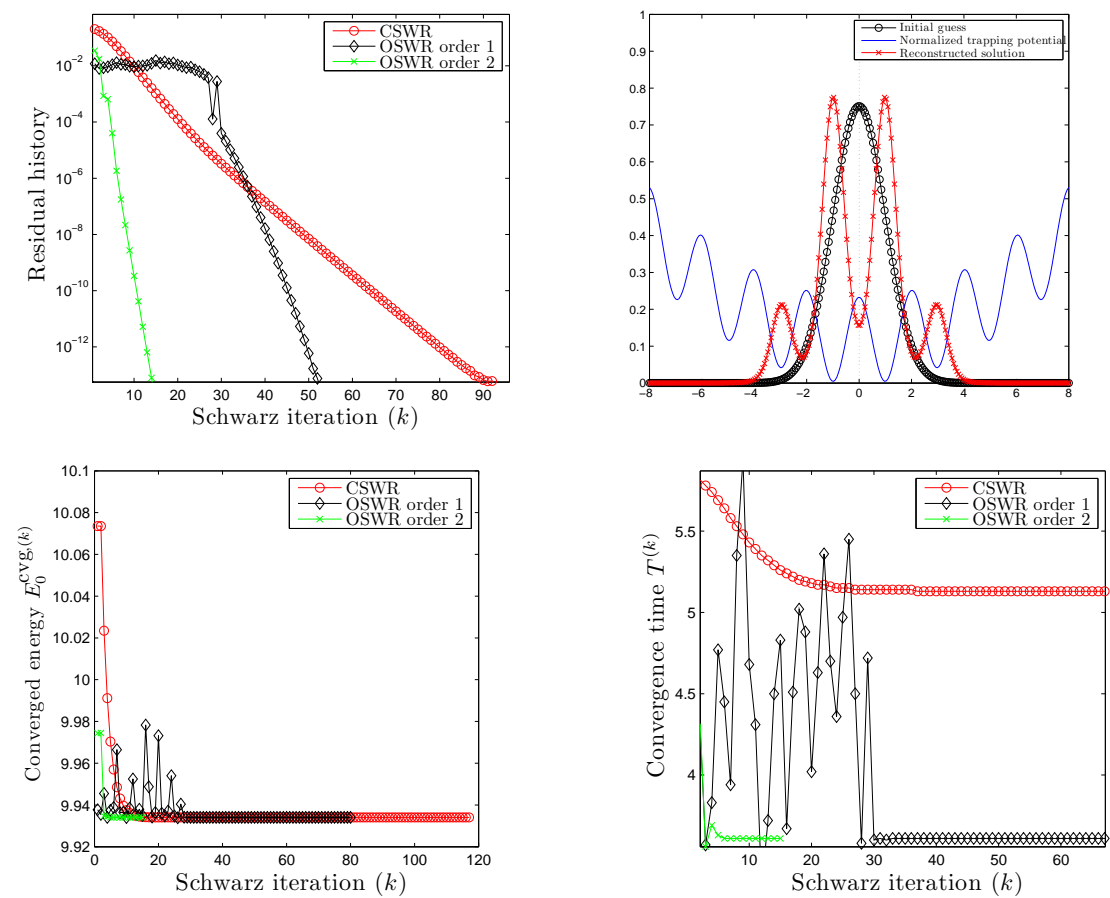

Figure 8: CSWR/OSWR DDMs: left (top): residual history. Right (top): converged solution (ground state). Left (bottom): total energy $E_{0}^{\mathrm{cvg},(k)}$ of the reconstructed CNGF converged solution $\phi^{\mathrm{cvg},(k)}$ vs. iteration $k$ until convergence. Right (bottom): stopping times $T^{(k)}$ vs. iteration $k$ until convergence.

\section{References}

[1] M. Al-Khaleel, A.E. Ruehli, and M.J. Gander. Optimized waveform relaxation methods for longitudinal partitioning of transmission lines. IEEE Transactions on Circuits and Systems, 56:1732-1743, 2009.

[2] X. Antoine, A. Arnold, C. Besse, M. Ehrhardt, and A. Schädle. A review of transparent and artificial boundary conditions techniques for linear and nonlinear Schrödinger equations. Commun. Comput. Phys., 4(4):729-796, 2008.

[3] X. Antoine, W. Bao, and C. Besse. Computational methods for the dynamics of the nonlinear Schrödinger/Gross-Pitaevskii equations. Comput. Phys. Comm., 184(12):2621-2633, 2013.

[4] X. Antoine and C. Besse. Construction, structure and asymptotic approximations of a microdifferential transparent boundary condition for the linear Schrödinger equation. J. Math. Pures Appl. (9), 80(7):701-738, 2001.

[5] X. Antoine, C. Besse, and S. Descombes. Artificial boundary conditions for one-dimensional cubic nonlinear Schrödinger equations. SIAM J. Numer. Anal., 43(6):2272-2293 (electronic), 2006.

[6] X. Antoine, C. Besse, and P. Klein. Absorbing boundary conditions for the one-dimensional 
Schrödinger equation with an exterior repulsive potential. J. Comput. Phys., 228(2):312-335, 2009 .

[7] X. Antoine, C. Besse, and P. Klein. Absorbing boundary conditions for general nonlinear Schrödinger equations. SIAM Journal on Scientific Computing, 33(2):1008-1033, 2011.

[8] X. Antoine, C. Besse, and J. Szeftel. Towards accurate artificial boundary conditions for nonlinear PDEs through examples. Cubo, 11(4):29-48, 2009.

[9] X. Antoine and R. Duboscq. GPELab, a Matlab toolbox to solve Gross-Pitaevskii equations I: Computation of stationary solutions. Computer Physics Communications, 185(11):2969-2991, 2014.

[10] X. Antoine and R. Duboscq. Robust and efficient preconditioned Krylov spectral solvers for computing the ground states of fast rotating and strongly interacting Bose-Einstein condensates. Journal of Computational Physics, 258C:509-523, 2014.

[11] X. Antoine and R. Duboscq. Modeling and computation of Bose-Einstein condensates: stationary states, nucleation, dynamics, stochasticity, volume 2146 of in Nonlinear Optical and Atomic Systems: at the Interface of Mathematics and Physics, CEMPI Subseries, 1st Volume, Lecture Notes in Mathematics. Springer, 2015.

[12] X. Antoine, E. Lorin, and A. Bandrauk. Domain decomposition method and high-order absorbing boundary conditions for the numerical simulation of the time dependent Schrödinger equation with ionization and recombination by intense electric field. Journal of Scientific Computing, 64(3):620-646, 2015.

[13] W. Bao. Ground states and dynamics of multicomponent Bose-Einstein condensates. Multiscale Modeling \& Simulation, 2(2):210-236, 2004.

[14] W. Bao and Y. Cai. Mathematical theory and numerical methods for Bose-Einstein condensation. Kinetic and Related Models, 6(1):1-135, 2013.

[15] W. Bao, I-L. Chern, and F.Y. Lim. Efficient and spectrally accurate numerical methods for computing ground and first excited states in Bose-Einstein condensates. Journal of Computational Physics, 219(2):836-854, 2006.

[16] W. Bao and Q. Du. Computing the ground state solution of Bose-Einstein condensates by a normalized gradient flow. SIAM J. Sci. Comput., 25(5):1674-1697, 2004.

[17] W. Bao and W. Tang. Ground-state solution of Bose-Einstein condensate by directly minimizing the energy functional. Journal of Computational Physics, 187(1):230-254, 2003.

[18] D. Baye and J-M. Sparenberg. Resolution of the Gross-Pitaevskii equation with the imaginarytime method on a Lagrange mesh. Physical Review E, 82(5):056701, 2010.

[19] C. Besse and F. Xing. Schwarz waveform relaxation method for one dimensional Schrödinger equation with general potential. submitted, 2015.

[20] M. L. Chiofalo, S. Succi, and M. P. Tosi. Ground state of trapped interacting Bose-Einstein condensates by an explicit imaginary-time algorithm. Physical Review E, 62(5):7438, 2000. 
[21] I. Danaila and F. Hecht. A finite element method with mesh adaptivity for computing vortex states in fast-rotating Bose-Einstein condensates. Journal of Computational Physics, 229(19):6946-6960, SEP 202010.

[22] I. Danaila and P. Kazemi. A new Sobolev gradient method for the direct computation of the Gross-Pitaevskii energy with rotation. SIAM Journal on Scientific Computing, 32(5):24472467, 2010.

[23] V. Dolean, P. Jolivet, and F. Nataf. An introduction to domain decomposition methods: theory and parallel implementation. 2015.

[24] M. Gander and L. Halpern. Optimized Schwarz waveform relaxation methods for advection reaction diffusion problems. SIAM J. Num. Anal., 45(2):666-697, 2007.

[25] M.J. Gander. Overlapping Schwarz for linear and nonlinear parabolic problems. In Proceedings of the 9th International Conference on Domain decomposition, pages 97-104, 1996.

[26] M.J. Gander. Optimal Schwarz waveform relaxation methods for the one-dimensional wave equation. SIAM J. Numer. Anal., 41:1643-1681, 2003.

[27] M.J. Gander. Optimized Schwarz methods. SIAM J. Numer. Anal., 44:699-731, 2006.

[28] M.J. Gander. Optimized Schwarz waveform relaxation methods for advection diffusion problems. SIAM J. Numer. Anal., pages 666-697, 2007.

[29] M.J. Gander, L. Halpern, and F. Nataf. Optimal convergence for overlapping and nonoverlapping Schwarz waveform relaxation. page 1999.

[30] M.J. Gander, F. Kwok, and B. Mandal. Dirichlet-Neumann and Neumann-Neumann waveform relaxation algorithms for parabolic problems. submitted, 2015.

[31] L. Halpern and J. Szeftel. Optimized and quasi-optimal Schwarz waveform relaxation for the one-dimensional Schrödinger equation. Math. Models Methods Appl. Sci., 20(12):2167-2199, 2010.

[32] R. Lascar. Propagation des singularités des solutions d'équations pseudo-différentielles quasi homogènes. Ann. Inst. Fourier (Grenoble), 27(2):vii-viii, 79-123, 1977.

[33] E. Lorin, X. Yang, and X. Antoine. Frozen gaussian approximation based domain decomposition methods for the linear and nonlinear Schrödinger equation beyond the semi-classical regime. submitted, 2015.

[34] B. Mandal. A time-dependent Dirichlet-Neumann method for the heat equation. page 2014.

[35] C. J. Pethick and H. Smith. Bose-Einstein condensation in dilute gases. Cambridge University Press, 2002.

[36] L. P. Pitaevskii and S. Stringari. Bose-Einstein condensation, volume 116. Clarendon press, 2003.

[37] R. Zeng and Y. Zhang. Efficiently computing vortex lattices in rapid rotating Bose-Einstein condensates. Computer Physics Communications, 180(6):854-860, 2009. 